\title{
One-dimensional chemotaxis kinetic model
}

\author{
Mohsen Sharifi tabar
}

\begin{abstract}
In this paper, we study a variation of the equations of a chemotaxis kinetic model and investigate it in one dimension. In fact, we use fractional diffusion for the chemoattractant in the Othmar-Dunbar-Alt system (Othmer in J Math Biol 26(3):263-298, 1988). This version was exhibited in Calvez in Amer Math Soc, pp 45-62, 2007 for the macroscopic well-known Keller-Segel model in all space dimensions. These two macroscopic and kinetic models are related as mentioned in Bournaveas, Ann Inst H Poincaré Anal Non Linéaire, 26(5):1871-1895, 2009, Chalub, Math Models Methods Appl Sci, 16(7 suppl):1173-1197, 2006, Chalub, Monatsh Math, 142(1-2):123-141, 2004, Chalub, Port Math (NS), 63(2):227-250, 2006. The model we study here behaves in a similar way to the original model in two dimensions with the spherical symmetry assumption on the initial data which is described in Bournaveas, Ann Inst H Poincaré Anal Non Linéaire, 26(5):1871-1895, 2009. We prove the existence and uniqueness of solutions for this model, as well as a convergence result for a family of numerical schemes. The advantage of this model is that numerical simulations can be easily done especially to track the blow-up phenomenon.
\end{abstract}

Mathematics Subject Classification (2000). Primary 92C17, 60J75;

Secondary 35L60, 92B05.

Keywords. Keller-Segel model, Othmar-Dunbar-Alt system, Chemotaxis, Kinetic model, Fractional diffusion, Hilbert transform, Numerical simulation.

\section{Introduction and main results}

Many models of biological cell movements involve a response of the cells to an external stimulus. Such responses are called taxis, which means to arrange. Different types of taxis are known, including aerotaxis, chemotaxis, geotaxis, haptotaxis [12]. Here we are interested in the case that the stimulus is a chemical substance (i.e. chemotaxis). In the case where the cells are large enough, they could sense the space gradient of the chemoattractant by measuring its 
density over their body and use this data to reorient in a more suitable manner. For an example of small cells and different types of motion, see [14]. This could be a positive response in the case where the chemical is a food or a signal from the other cells to absorb each other, or negative response in the case where they want to move away from a noxious substance. In the absence of external cues, many organisms use a random walk strategy to determine their pattern of movement. So one of the simplest way to describe this motion is given by the flux

$$
j=-D \nabla n+n u_{c}
$$

where $n$ is the cells density and $u_{c}$ is the macroscopic chemotactic velocity. Keller and Segel [19] postulated that the chemotactic velocity is given by $u_{c}=\chi(S) \nabla S$ where $S$ is the density of chemical substance and $\chi(S)$ is called the chemotactic sensitivity. By assuming that there exists no cell division or death, one has the classical conservation model

$$
\partial_{t} n=\nabla \cdot(D \nabla n-n \chi(S) \nabla S) .
$$

To achieve a complete system of equations, (1.1) must be coupled with an evolution equation for chemical substance and possibly other variables (except when the density of the chemical substance is predefined).

This macroscopic model has been investigated by many authors (e.g. see $[2,6,11,18])$. If one looks at the kinetic level, a good model is the so-called Othmar-Dunbar-Alt system [20] which is based on velocity-jump processes and leads to the transport equation

$$
\partial_{t} f+v . \nabla_{x} f=\int_{v^{\prime} \in V} T[S]\left(t, x, v^{\prime} \mapsto v\right) f\left(t, x, v^{\prime}\right) \mathrm{d} v^{\prime}-\lambda[S](t, v, x) f(t, v, x),
$$

for $v \in V \subset \mathbb{R}^{d}$ where $f(t, x, v)$ denotes the cellular density. The turning kernel $T[S]\left(t, x, v^{\prime} \mapsto v\right)$ denotes the probability of transition from velocity $v^{\prime}$ to $v$, and

$$
\lambda[S]=\int_{v^{\prime} \in V} T[S]\left(t, x, v \mapsto v^{\prime}\right) \mathrm{d} v^{\prime},
$$

is the intensity of this Poisson process. The influence of $S$, the density of the chemical substance, is highlighted in the notation $T[S]$. One could follow this model in $[3-5,8-10,13,15-17,21]$.

We are interested in the particular choice

$$
T[S]\left(t, x, v^{\prime} \mapsto v\right)=\chi[v \cdot \nabla S(t, x)]_{+},
$$

with constant $\chi>0$, that is to say that cells choose only favorable directions when they reorientate, and they align more likely with the gradient of the chemical. Here for $z \in \mathbb{R}$ we let

$$
[z]_{+}=\max \{z, 0\}, \quad[z]_{-}=\max \{-z, 0\} .
$$


So with this choice of $T$, the system (1.2) in one dimension (i.e. $x \in \mathbb{R}, v \in$ $V \subset \mathbb{R}$ ) reads

$\partial_{t} f+v \partial_{x} f=\chi\left[v \partial_{x} S(t, x)\right]_{+} n(t, x)-\chi f(t, v, x) \int_{v^{\prime} \in V}\left[v^{\prime} \partial_{x} S(t, x)\right]_{+} \mathrm{d} v^{\prime}$,

where

$$
n(t, x)=\int_{v \in V} f(t, v, x) \mathrm{d} v,
$$

is the cells density.

As we mentioned above, to achieve a complete system of equations, we must consider the evolution of the chemical. There are many choices for the evolution of the chemoattractant, one of them is to use a parabolic diffusion equation, (e.g. see $[6,8,10,11,17,21]$ )

$$
\varepsilon \partial_{t} S-\Delta S+\alpha S=n
$$

$\alpha \geq 0$ is the chemical degradation. By assuming a very fast diffusion of the chemical substance, one could use the following elliptic equation, (e.g. see $[2,4,5,9,17])$

$$
-\Delta S+\alpha S=n
$$

In the case of the classical Keller-Segel model, using equations (1.1) and (1.5) to describe the cells density has a good, and experimentally validated, result in two dimensions: There exists a critical mass $M_{\text {crit }}$ and if the initial mass of cells is below this mass, there is a global in time solution to this system, and above this mass, the solutions will blow-up in finite time (see $[2,18]$ ). For higher dimensions, there is no more a critical mass, i.e. the $L^{1}$-norm does not play a crucial role and blow-up may occur with any initial mass. As a matter of fact, the significant space is $L^{d / 2}$. (see [11]).

In [7], the authors proposed a variation of the classical model which behaves in this critical manner in all dimensions: to change the equation of chemoattractant by the convolution of a logarithmic kernel with the cells density. In dimension two, this is equivalent to the above mentioned model. In one dimension, this is equivalent to use a fractional diffusion for the chemoattractant. The advantage of this model is that numerical simulations could be easily made in one dimension.

We choose the same variation for Othmar-Dunbar-Alt system. We consider the problem in both spaces $X=\mathbb{R}$, the whole space, and $X=S^{1}$, the circle (i.e. a periodic medium). Let $V \subset \mathbb{R}$ be a finite set of velocities and let

$$
\mu_{+}=\sum_{v>0} v, \quad \mu_{-}=-\sum_{v<0} v
$$

The reason to choose a finite set of velocities is to simplify the model to handle it in a simpler framework. One should notice that it is not a serious restriction 
as we will see in remark 3.7. We end up with the following equations in one dimension,

$$
\left\{\begin{array}{l}
\partial_{t} f+v \partial_{x} f=\left[v \partial_{x} S\right]_{+} n-\left(\mu_{+}\left[\partial_{x} S\right]_{+}+\mu_{-}\left[\partial_{x} S\right]_{-}\right) f \\
(-\Delta)^{\frac{1}{2}} S+\alpha S=n
\end{array}\right.
$$

We will clarify the notation $(-\Delta)^{\frac{1}{2}}$ in definition 2.1 . Note that one could multiply the densities by $\chi$ and so we may assume that $\chi=1$ without loss of generality.

The natural and usual question of blow-up arises here: Is there any aggregation between cells that could overcome the transport of the particles? Note that since in the system (1.7) the total mass is formally conserved, one may expect the aggregation of cells to form a blow-up. Before answering to this question, we need an existence result for this system, actually at this point in the usual Sobolev spaces,

Proposition 1.1. If the initial data $f_{0}(v, x)$ is in the space $L^{1}\left(V ; H^{s}\right)$ and $\frac{1}{2}<$ $s \leq 1$, there exists a local in time solution to the problem (1.7) in the space $C\left([0, T] ; L^{1}\left(V ; H^{s}\right)\right)$ with $f(0, v, x)=f_{0}(v, x)$ and this solution is unique.

Once we know about the existence of the solution, we may ask about the conditions for blow-up. We are not able to answer this question theoretically here and so we try to develop numerical methods to simulate the problem. As usual, it seems that one needs large mass for blow-up to occur. In the whole space, we also need to prevent the cells to spread in the space. Technically it seems that some moments of the initial data should be small.

The outline of this paper is as follows. In Sect. 2, we introduce the notations which are used throughout this paper and prove a few general lemmas, some of them are of own interest. The next section concerns a local-in-time existence result for this system and we develop some theorem to find estimates on the solution and its lifetime. We prove also the conservation of mass and nonnegativity of initial data. The paper proceeds in Sect. 4 with the presentation of numerical methods for which we prove convergence of the numerical scheme. In Sect. 5, We investigate the blow-up phenomenon and some results from the implementation of numerical schemes are presented.

\section{Notations and preliminary results}

In this section, we introduce a few notations which are used throughout this paper. Moreover we mention some estimates that we use in what follows.

Here we recall the definitions of the Fourier and Hilbert transforms.

We use $\mathbb{R} / \mathbb{Z}$ for the circle in this article and take the interval $(-1 / 2,1 / 2]$ as its representation. By the notation $L^{p}, 1 \leq p \leq \infty$, we mean either $L^{p}(\mathbb{R})$ or $L^{p}(\mathbb{R} / \mathbb{Z}) .\|\varphi\|_{p}$ stands for the $L^{p}$-norm of the function $\varphi \in L^{p}$ and

$$
\|(\varphi, \psi)\|_{2}=\left(\|\varphi\|_{2}^{2}+\|\psi\|_{2}^{2}\right)^{1 / 2}, \quad\|(\varphi, \psi)\|_{\infty}=\max \left\{\|\varphi\|_{\infty},\|\psi\|_{\infty}\right\} .
$$


The Fourier transform $\mathcal{F}: L^{2}(\mathbb{R}) \rightarrow L^{2}(\mathbb{R})$ is defined as

$$
\mathcal{F} \varphi(\xi)=\widehat{\varphi}(\xi)=\int_{-\infty}^{\infty} \mathrm{e}^{-2 \pi \mathrm{i} \xi x} \varphi(x) \mathrm{d} x, \quad \xi \in \mathbb{R} .
$$

Also $\mathcal{F}: L^{2}(\mathbb{R} / \mathbb{Z}) \rightarrow \ell^{2}(\mathbb{Z})$ is defined as

$$
\mathcal{F} \varphi(\xi)=\widehat{\varphi}(\xi)=\int_{-\frac{1}{2}}^{\frac{1}{2}} \mathrm{e}^{-2 \pi \mathrm{i} \xi x} \varphi(x) \mathrm{d} x, \quad \xi \in \mathbb{Z} .
$$

Definition 2.1. By $(-\Delta)^{\frac{1}{2}}$ we denote the transformation whose Fourier symbol is $2 \pi|\xi|$, i.e.

$$
\mathcal{F}\left((-\Delta)^{\frac{1}{2}} \varphi\right)(\xi)=2 \pi|\xi| \mathcal{F} \varphi(\xi), \quad \text { for almost every } \xi \in \mathbb{R} \text {, or } \xi \in \mathbb{Z} .
$$

The above definition is related to the well-known Hilbert transform $\mathbb{H}$ : $L^{2}(\mathbb{R}) \rightarrow L^{2}(\mathbb{R})$ which is defined as

$$
\mathbb{H} \varphi(x)=\frac{1}{\pi} \text { p.v. } \int_{-\infty}^{\infty} \frac{\varphi(y)}{x-y} \mathrm{~d} y, \quad \text { for almost every } x \in \mathbb{R} .
$$

The Hilbert transform is an isomorphism on $L^{2}(\mathbb{R})$ and using Fourier transform, one may define it as

$$
\mathcal{F}(\mathbb{H} \varphi)(\xi)=-\mathrm{i} \operatorname{sgn}(\xi) \mathcal{F} \varphi(\xi) .
$$

This may be used to define the Hilbert transform on $L^{2}(\mathbb{R} / \mathbb{Z})$ which can be written in the integral form as

$$
\mathbb{H} \varphi(x)=\text { p.v. } \int_{-\frac{1}{2}}^{\frac{1}{2}} \cot \pi(x-y) \varphi(y) \mathrm{d} y,
$$

upon using $\cot (\pi x)=\sum_{\xi \in \mathbb{Z}}-\mathrm{i} \operatorname{sgn}(\xi) \mathrm{e}^{2 \pi \mathrm{i} \xi x}$ in the distribution sense.

Definition 2.2. For $\alpha>0$, the generalized Hilbert transform $\mathbb{H}_{\alpha}$ is defined as follows,

$$
\mathcal{F}\left(\mathbb{H}_{\alpha} \varphi\right)(\xi)=\frac{-2 \pi \mathrm{i} \xi}{\alpha+2 \pi|\xi|} \mathcal{F} \varphi(\xi) .
$$

For a fixed $\alpha$, if there is no ambiguity, we denote $\mathcal{H}:=\mathbb{H}_{\alpha}$ for simplicity.

By this definition, one might rewrite the equation concerning the chemical substance in system (1.7) as

$$
\partial_{x} S=-\mathcal{H} n,
$$

and so (1.7) is reduced to

$$
\partial_{t} f+v \partial_{x} f=[v \mathcal{H} n]_{-} n-\left(\mu_{+}[\mathcal{H} n]_{-}+\mu_{-}[\mathcal{H} n]_{+}\right) f .
$$

To find a solution to this problem, we need to find a space in which the nonlinearity behaves nicely. Since the nonlinearity is quadratic and the Hilbert transform does not act nicely on $L^{\infty}$, It seems that the best choice is to work with the Sobolov spaces. 
By the notaion $H^{s}$, we mean the usual fractional Sobolev space in either $\mathbb{R}$ or $\mathbb{R} / \mathbb{Z}$ with the norm

$$
\|\varphi\|_{H^{s}}=\left\|\left(1+4 \pi^{2}|\xi|^{2}\right)^{s / 2} \mathcal{F} \varphi(\xi)\right\|_{2}
$$

where in the case of $\mathbb{R} / \mathbb{Z},\|\cdot\|_{2}$ means the norm of $\ell^{2}(\mathbb{Z})$.

In one dimension for $s>1 / 2$, this space is embedded in $L^{\infty}$ and as a consequence it is closed under the point-wise multiplication. Also it is obviously mapped into itself by the mapping $\mathcal{H}$. In fact we may use more accurate estimates in the case $s=1$.

Lemma 2.3. For any $\varphi \in H^{1}(\mathbb{R})$,

$$
\begin{gathered}
\max \left\{\|\varphi\|_{\infty},\|\mathcal{H} \varphi\|_{\infty}\right\} \leq\|\varphi\|_{2}^{1 / 2}\left\|\partial_{x} \varphi\right\|_{2}^{1 / 2} \\
\leq\|\varphi\|_{1}^{1 / 3}\left\|\partial_{x} \varphi\right\|_{2}^{2 / 3} .
\end{gathered}
$$

Proof.

$$
\begin{aligned}
\|\varphi\|_{\infty} & \leq\|\mathcal{F} \varphi\|_{1} \\
& \leq\left\|\left(1+4 \varepsilon^{2} \pi^{2} \xi^{2}\right)^{-\frac{1}{2}}\right\|_{2}\left\|\left(1+4 \varepsilon^{2} \pi^{2} \xi^{2}\right)^{\frac{1}{2}} \mathcal{F} \varphi(\xi)\right\|_{2} \\
& =\sqrt{\frac{1}{2 \varepsilon}\left(\|\varphi\|_{2}^{2}+\varepsilon^{2}\left\|\partial_{x} \varphi\right\|_{2}^{2}\right)} .
\end{aligned}
$$

Choose $\varepsilon=\|\varphi\|_{2} /\left\|\partial_{x} \varphi\right\|_{2}$. The estimate for $\mathcal{H} \varphi$ is exactly the same. Now

$$
\begin{aligned}
\|\varphi\|_{2}^{2} & =\|\mathcal{F} \varphi\|_{2}^{2} \\
& \leq\|\mathcal{F} \varphi\|_{\infty}\|\mathcal{F} \varphi\|_{1} \\
& \leq\|\varphi\|_{1}\left(\|\varphi\|_{2}\left\|\partial_{x} \varphi\right\|_{2}\right)^{1 / 2} .
\end{aligned}
$$

Therefore

$$
\|\varphi\|_{2} \leq\|\varphi\|_{1}^{2 / 3}\left\|\partial_{x} \varphi\right\|_{2}^{1 / 3}
$$

which proves (2.4).

Remark 2.4. The constant in the estimate (2.3) is the best. Choose $\varphi(x)=$ $C \mathrm{e}^{-\lambda\left|x-x_{0}\right|}$. In fact this family is the only functions in $H^{1}(\mathbb{R})$ for whom the equality occurs. To see this, note that the required condition for the second estimate to be an equality is $|\mathcal{F} \varphi(\xi)|=C /\left(1+4 \pi^{2} \varepsilon^{2} \xi^{2}\right)$, i.e

$$
\mathcal{F} \varphi(\xi)=\frac{C \mathrm{e}^{2 \pi \mathrm{i} \theta_{\xi}}}{1+4 \pi^{2} \varepsilon^{2} \xi^{2}} .
$$

For the first estimate not to be strict, we need that the equality occur at some point $x_{0}$,

$$
\left|\varphi\left(x_{0}\right)\right|=\int|\mathcal{F} \varphi(\xi)| \mathrm{d} \xi=\int \frac{|C|}{1+4 \pi^{2} \varepsilon^{2} \xi^{2}} \mathrm{~d} \xi .
$$

On the other hand,

$$
\varphi\left(x_{0}\right)=\int \mathrm{e}^{2 \pi \mathrm{i} x_{0} \xi} \mathcal{F} \varphi(\xi) \mathrm{d} \xi=\int \frac{C \mathrm{e}^{2 \pi \mathrm{i}\left(x_{0} \xi+\theta_{\xi}\right)}}{1+4 \pi^{2} \varepsilon^{2} \xi^{2}} \mathrm{~d} \xi,
$$


which implies

$$
\int \frac{1}{1+4 \pi^{2} \varepsilon^{2} \xi^{2}} \mathrm{~d} \xi=\left|\int \frac{\mathrm{e}^{2 \pi \mathrm{i}\left(x_{0} \xi+\theta_{\xi}\right)}}{1+4 \pi^{2} \varepsilon^{2} \xi^{2}} \mathrm{~d} \xi\right|,
$$

and this is the case only when $\theta_{\xi}=\theta_{0}-x_{0} \xi$. After normalizing $\varphi$, it is the only weak solution of the problem $\varphi-\varepsilon^{2} \Delta \varphi=\delta_{x_{0}}$ in $H^{1}(\mathbb{R})$, i.e. $\varphi(x)=$ $C \mathrm{e}^{-\varepsilon^{-1}\left|x-x_{0}\right|}$. Continuing this argument, we conclude that the best possible constant in (2.4) is less than 1 , but is not less than $2^{-1 / 3}$. Another consequence is that if we define

$$
J(\varphi)=\|\varphi\|_{\infty}, \quad \varphi \in H^{1}(\mathbb{R})
$$

then

$$
\max _{\|\varphi\|_{H^{1}}=1} J(\varphi)=\frac{1}{\sqrt{2}}=J\left(\varphi_{x_{0}}^{ \pm}\right)
$$

where

$$
\varphi_{x_{0}}^{ \pm}(x)= \pm \frac{1}{\sqrt{2}} \mathrm{e}^{-\left|x-x_{0}\right|}
$$

and if $\|\varphi\|_{H^{1}}=1$ and $J(\varphi)=1 / \sqrt{2}$, one has $\varphi=\varphi_{x_{0}}^{ \pm}$for some $x_{0} \in \mathbb{R}$.

Remark 2.5. These inequalities holds true for the circle if we replace $\varphi$ by $\varphi-\langle\varphi\rangle$, i.e.

$$
\max \left\{\|\varphi-\langle\varphi\rangle\|_{\infty},\|\mathcal{H} \varphi\|_{\infty}\right\} \leq\|\varphi-\langle\varphi\rangle\|_{2}^{1 / 2}\left\|\partial_{x} \varphi\right\|_{2}^{1 / 2} \leq\|\varphi\|_{2}^{1 / 2}\left\|\partial_{x} \varphi\right\|_{2}^{1 / 2}
$$

where $\langle\varphi\rangle=\mathcal{F} \varphi(0)$ is the mean value of $\varphi$ over the circle. The proof is essentially the same, using the fact that the zero component of the Fourier transform is zero. Note that this is automatically the case for $\mathcal{H} \varphi$.

On the circle, we have similar estimate which involves only the norm of the derivative,

Lemma 2.6. For any $\varphi \in H^{1}(\mathbb{R} / \mathbb{Z})$,

$$
\max \left\{\|\varphi-\langle\varphi\rangle\|_{\infty},\|\mathcal{H} \varphi\|_{\infty}\right\} \leq \frac{1}{2 \sqrt{3}}\left\|\partial_{x} \varphi\right\|_{2} .
$$

Proof.

$$
\begin{aligned}
\|\varphi-\langle\varphi\rangle\|_{\infty} & \leq \sum_{\substack{\xi \in \mathbb{Z} \\
\xi \neq 0}}|\mathcal{F} \varphi(\xi)| \\
& \leq\left(\sum_{\xi \neq 0} \frac{1}{4 \pi^{2} \xi^{2}}\right)^{\frac{1}{2}}\left(\sum_{\xi \neq 0} 4 \pi^{2} \xi^{2}|\mathcal{F} \varphi(\xi)|^{2}\right)^{\frac{1}{2}} \\
& =\frac{1}{2 \sqrt{3}}\left\|\partial_{x} \varphi\right\|_{2} .
\end{aligned}
$$

It is clear that the same estimate holds for $\mathcal{H} \varphi$. 
Remark 2.7. Again the equality may occur. Choose $\varphi(x)=x^{2}$ for $x \in\left(-\frac{1}{2}, \frac{1}{2}\right]$. In fact a similar argument as remark (2.4) proves that if we define

$$
J(\psi)=\|\psi\|_{\infty}, \quad \psi \in H^{1}(\mathbb{R} / \mathbb{Z})
$$

then

$$
\max _{\substack{\left\|\partial_{x} \psi\right\|_{2}=1 \\\langle\psi\rangle=0}} J(\psi)=\frac{1}{2 \sqrt{3}}=J\left(\psi_{x_{0}}^{ \pm}\right)
$$

where

$$
\psi_{x_{0}}^{ \pm}(x)= \pm \sqrt{3}\left(\frac{1}{12}-\left(x-x_{0}\right)^{2}\right),
$$

and if $\left\|\partial_{x} \psi\right\|_{2}=1,\langle\psi\rangle=0$ and $J(\psi)=1 / \sqrt{2}$, one has $\psi=\psi_{x_{0}}^{ \pm}$for some $x_{0} \in \mathbb{R} / \mathbb{Z}$.

In the following we assume that $1 / 2<s \leq 1$. The notation $A \lesssim B$ means that there exists a "universal positive constant" $\mathcal{C}$ such that $A \leq \mathcal{C} B$, where by "universal" we mean that $\mathcal{C}$ does not depend on some of the parameters which will be clarified in each context.

Lemma 2.8. For any $\varphi, \psi \in H^{s}$,

$$
\begin{gathered}
\|\mathcal{H} \varphi\|_{H^{s}} \leq\|\varphi\|_{H^{s}} \\
\max \left\{\|\varphi\|_{\infty},\|\mathcal{H} \varphi\|_{\infty}\right\} \lesssim\|\varphi\|_{H^{s}}, \\
\|\varphi \psi\|_{H^{s}} \lesssim\|\varphi\|_{H^{s}}\|\psi\|_{H^{s}} \\
|\varphi(x)-\varphi(y)| \lesssim\|\varphi\|_{H^{s}}|x-y|^{s-1 / 2} .
\end{gathered}
$$

Proof. (2.6) is clear and (2.7) is proved using the same technique as lemmas (2.3) and (2.6). The optimized value of the constants are different in the two cases $\mathbb{R}$ or $\mathbb{R} / \mathbb{Z}$. Although (2.8) and (2.9) is classic, for the reader's convenience, we recall briefly how they can be obtained. For (2.8), one has

$$
\begin{aligned}
|\xi|^{s}|\widehat{\varphi \psi}(\xi)| & =|\xi|^{s}|\widehat{\varphi} * \widehat{\psi}(\xi)| \\
& \leq|\xi|^{s} \int|\widehat{\varphi}(\xi-\zeta) \widehat{\psi}(\zeta)| \mathrm{d} \zeta \\
& \leq \int\left(|\xi-\zeta|^{s}+|\zeta|^{s}\right)|\widehat{\varphi}(\xi-\zeta) \widehat{\psi}(\zeta)| \mathrm{d} \zeta \\
& =\left(|\xi|^{s}|\widehat{\varphi}|\right) *|\widehat{\psi}|(\xi)+\left(|\xi|^{s}|\widehat{\psi}|\right) *|\widehat{\varphi}|(\xi),
\end{aligned}
$$

and since $\widehat{\varphi}$ and $\widehat{\psi}$ are absolutely integrable, so $|\xi|^{s}|\widehat{\varphi \psi}(\xi)|$ is square integrable. To prove (2.9), one has

$$
\begin{aligned}
& \|\varphi(\cdot+h)-\varphi(\cdot)\|_{\infty} \leq\left\|\left(\mathrm{e}^{2 \pi \mathrm{i} h \xi}-1\right) \mathcal{F} \varphi(\xi)\right\|_{1} \\
& \quad \leq\left\|\frac{\mathrm{e}^{2 \pi \mathrm{i} h \xi}-1}{\left(1+4 \pi^{2} \xi^{2}\right)^{s / 2}}\right\|_{2}\|\varphi\|_{H^{s}} .
\end{aligned}
$$

One could divide the integration in two parts $|\xi|>h^{-1}$ and $|\xi|<h^{-1}$ to complete the proof. 
Definition 2.9. For a fixed $1 / 2<s \leq 1$, we denote $H:=L^{1}\left(V ; H^{s}\right)$. Note that $H$ is isomorphic to the product of $\operatorname{card}(V)$-times the space $H^{s}$ and if $\mathbf{f}_{v}$, $v \in V$, denotes the components of $\mathbf{f} \in H$, then $H$ is endowed with the norm

$$
\|\mathbf{f}\|_{H}=\sum_{v \in V}\left\|\mathbf{f}_{v}\right\|_{H^{s}} .
$$

We define the space $L=L^{1}\left(V ; L^{2}\right)$ in a similar way.

Definition 2.10. We denote the nonlinearity in (2.2) by $\mathbf{N}$, i.e. $\mathbf{N}: H \rightarrow H$ is defined by

$$
(\mathbf{N f})_{v}=[v \mathcal{H} n]_{-} n-\left(\mu_{+}[\mathcal{H} n]_{-}+\mu_{-}[\mathcal{H} n]_{+}\right) \mathbf{f}_{v},
$$

where $\mathbf{f} \in H$ and $n=\sum_{v \in V} \mathbf{f}_{v}$.

The fact that $\mathbf{N}$ maps $H$ to $H$, is a consequence of two points. Firstly, the point-wise multiplication maps $H^{s}$ to itself (continuously) using (2.8). Secondly, the operator $\varphi \mapsto[\varphi]_{+}$maps $H^{s}$ to itself. As Luc Tartar pointed out, it can be seen by an intrinsic norm of the $H^{s}\left(\mathbb{R}^{d}\right)$ for $0<s<1$ which is defined by

$$
\|\varphi\|_{H^{s}}^{2}=\|\varphi\|_{2}^{2}+\iint \frac{|\varphi(x)-\varphi(y)|^{2}}{|x-y|^{d+2 s}} \mathrm{~d} x \mathrm{~d} y,
$$

(see for instance [1]). In fact we can state the following lemma (we thank Otared Kavian for the proof in the case $0<s<1$ ),

Lemma 2.11. The mapping $\varphi \mapsto[\varphi]_{+}$is continuous on $H^{s}$ for $0<s \leq 1$ and hence $\mathbf{N}$ is also continuous on $H$.

Proof. We consider two cases $s=1$ and $0<s<1$ separately. For the case $s=1$, First let $\varphi \in H^{1}(X)$. Since $\varphi$ is continuous, the open set $\{\varphi>0\}$ is equal to at most countable union of nonintersecting open intervals $\left(a_{i}, b_{i}\right)$ and $\varphi$ is zero at endpoints. So it is easy by using test functions to check that the week derivative of $[\varphi]_{+}$exist and equals to $\partial_{x} \varphi \mathbf{1}_{\{\varphi>0\}}$ and this function is obviously square integrable. Similarly $\partial_{x}[\varphi]_{-}=-\partial_{x} \varphi \mathbf{1}_{\{\varphi<0\}}$ and it is concluded that

$$
\partial_{x} \varphi \mathbf{1}_{\{\varphi=0\}}=\partial_{x} \varphi-\partial_{x}[\varphi]_{+}+\partial_{x}[\varphi]_{-}=\partial_{x} \varphi-\partial_{x}\left([\varphi]_{+}-[\varphi]_{-}\right)=0 .
$$

Now let $\varphi_{k} \rightarrow \varphi$ in $H^{1}$ and choose any $\varepsilon>0$. Since

$$
\{\varphi=0\}=\bigcap_{m \geq 1}\{|\varphi|<1 / m\},
$$

$\partial_{x} \varphi \mathbf{1}_{\{\varphi=0\}}=0$ and $\partial_{x} \varphi \in L^{2}$, Lesbesgue's dominated convergence theorem implies that $\int\left|\partial_{x} \varphi\right|^{2} \mathbf{1}_{\{|\varphi|<1 / m\}} \rightarrow 0$ as $m \rightarrow \infty$. So one may choose $\delta>0$ such that $\int\left|\partial_{x} \varphi\right|^{2} \mathbf{1}_{\{|\varphi|<\delta\}}<\varepsilon$. Now for $k$ large enough, $\int\left|\partial_{x} \varphi_{k}-\partial_{x} \varphi\right|^{2}<\varepsilon$ and $\left\|\varphi_{k}-\varphi\right\|_{\infty}<\delta$ which completes the proof since on the set $\{|\varphi| \geq \delta\}$ both $\varphi_{k}$ and $\varphi$ have the same sign and on the set $\{|\varphi|<\delta\}$ we have control on both $\left\|\partial_{x} \varphi_{k}-\partial_{x} \varphi\right\|_{2}$ and $\left\|\partial_{x} \varphi_{k}+\partial_{x} \varphi\right\|_{2}$.

For $0<s<1$, we use the very definition of the norm on the space $H^{s}$. Let $\varphi_{k} \rightarrow \varphi$ in $H^{s}$. Assume by the way of contradiction that $f_{k}=\left[\varphi_{k}\right]_{+}-[\varphi]_{+}$ 
does not converge to 0 in $H^{s}$. After passing to subsequences, we may assume that $\left\|f_{k}\right\|_{H^{s}} \geq \varepsilon>0$ and also $\varphi_{k}-\varphi$ tends to zero almost everywhere. Since

$$
\left|[a]_{+}-[b]_{+}\right| \leq|a-b|,
$$

for any real numbers $a, b$, we conclude that $f_{k} \rightarrow 0$ almost everywhere and

$$
\begin{aligned}
\left|f_{k}(x)-f_{k}(y)\right|^{2} & \leq 2\left(\left|\left[\varphi_{k}(x)\right]_{+}-\left[\varphi_{k}(y)\right]_{+}\right|^{2}+\left|[\varphi(x)]_{+}-[\varphi(y)]_{+}\right|^{2}\right) \\
& \leq 2\left(\left|\varphi_{k}(x)-\varphi_{k}(y)\right|^{2}+|\varphi(x)-\varphi(y)|^{2}\right) .
\end{aligned}
$$

Let $\mathrm{d} \mu=|x-y|^{-(1+2 s)} \mathrm{d} x \mathrm{~d} y$. From $\left\|\varphi_{k}-\varphi\right\|_{H^{s}} \rightarrow 0$ and the very definition of the norm of space $H^{s}$, we have $\varphi_{k}(x)-\varphi_{k}(y) \longrightarrow \varphi(x)-\varphi(y)$ in $L^{2}(\mathrm{~d} \mu)$. Hence again after passing to a subsequence, we may dominate the functions $\varphi_{k}(x)-\varphi_{k}(y)$ by a function $G$ in $L^{2}(\mathrm{~d} \mu)$. Therefore we may apply the Lesbesgue's dominated convergence theorem to the sequence $\left\{f_{k}(x)-f_{k}(y)\right\}_{k}$ to conclude that this sequence converges to zero in $L^{2}(\mathrm{~d} \mu)$, i.e. $\left\|f_{k}\right\|_{H^{s}} \rightarrow 0$, a contradiction.

Remark 2.12. Note that the first part of this result does not depend on dimension of the space and it is true in all dimensions, although we need to be in one dimension and to have $s>1 / 2$ for $\mathbf{N}$ to be continuous. See also remark (3.6)

Remark 2.13. The truncation map is not locally Lipschitz on $H^{s}$, for $1 / 2<s$ $\leq 1$. To see this, let $\varphi \in H^{s}$ to be identically zero on a small interval $\left(x_{0}, x_{1}\right)$. Let $\psi \in H^{s}$ to be a cut-off function for some subinterval $\left(x_{0}^{\prime}, x_{1}^{\prime}\right)$ which is defined by

$$
\psi(x)=\frac{x-x_{0}}{x_{0}^{\prime}-x_{0}} \mathbf{1}_{\left(x_{0}, x_{0}^{\prime}\right)}+\mathbf{1}_{\left(x_{0}^{\prime}, x_{1}^{\prime}\right)}+\frac{x_{1}-x}{x_{1}-x_{1}^{\prime}} \mathbf{1}_{\left(x_{1}^{\prime}, x_{1}\right)},
$$

and let $\psi_{k}(x)=k^{-\gamma} \psi(x)$. Now consider

$$
\begin{aligned}
& f_{k}(x)=\varphi(x)+\left(1+\cos 2 \pi k \frac{x-x_{0}}{x_{1}-x_{0}}\right) \psi_{k}(x), \\
& g_{k}(x)=\varphi(x)+\left(-1+\cos 2 \pi k \frac{x-x_{0}}{x_{1}-x_{0}}\right) \psi_{k}(x) .
\end{aligned}
$$

So $f_{k} \geq 0, g_{k} \leq 0$ and hence

$$
\left\|\left[f_{k}\right]_{+}-\left[g_{k}\right]_{+}\right\| H_{H^{s}}=\left\|f_{k}\right\|_{H^{s}} \gtrsim k^{-\gamma+s-1 / 2},
$$

using the Hölder estimate (2.9). But

$$
\left\|f_{k}-g_{k}\right\|_{H^{s}}=2\left\|\psi_{k}\right\|_{H^{s}}=2\|\psi\|_{H^{s}} k^{-\gamma} .
$$

We may choose $\gamma$ large enough such that $\left\|f_{k}-\varphi\right\|_{H^{s}}+\left\|g_{k}-\varphi\right\|_{H^{s}} \rightarrow 0$. In fact one may choose any $\gamma>s-1 / 2$. So the truncation map is not locally Lipschitz around $\varphi$ and hence around the closure of such functions in $H^{s}$ and it is not difficult to see that any function in $H^{s}$ which vanishes at some point is in this closure.

Definition 2.14. The shift operator is defined as $\mathcal{S}_{y} \varphi(x)=\varphi(x-y)$. Also define the transport group $\mathbf{S}_{t}$ for $t \in \mathbb{R}$ on the space $H$ or $L$ as

$$
\left(\mathbf{S}_{t} \mathbf{f}\right)_{v}=\mathcal{S}_{v t} \mathbf{f}_{v} .
$$


It is easy to see that the generalized Hilbert transform commutes with the shift operator and $\mathcal{S}_{y}$ is an isometry on $L^{2}$ or $H^{s}$ since

$$
\mathcal{F}\left(\mathcal{S}_{y} \varphi\right)(\xi)=\mathrm{e}^{-2 \pi \mathrm{i} y \xi} \mathcal{F} \varphi(\xi) .
$$

As a consequence, $\mathbf{S}_{t}$ is an isometry on $H$ or $L$. Also, clearly, for a fixed $\mathbf{f} \in H$ or $\mathbf{f} \in L$ the mapping $t \mapsto \mathbf{S}_{t} \mathbf{f}$ is continuous, i.e. $\mathbf{S}_{t}$ is a $C^{0}$-group of isometries acting on $H$ or $L$. As a consequence if $\mathbf{f} \in C([0, T] ; H)($ resp. $\mathbf{f} \in C([0, T] ; L))$, then $t \mapsto \mathbf{S}_{t} \mathbf{f}(t, \cdot)$ belongs to $C([0, T] ; H)(\operatorname{resp} . C([0, T] ; L))$.

Using the group $\mathbf{S}_{t}$ and the operator $\mathbf{N}$ introduced in (2.10), it is reasonable to rewrite $(2.2)$ as

$$
\partial_{t} \mathbf{f}_{\star}=\mathbf{S}_{-t} \mathbf{N} \mathbf{S}_{t} \mathbf{f}_{\star},
$$

where for $\mathbf{f}:[0, T] \rightarrow H$ or $L$, we denote

$$
\mathbf{f}_{\star}(t, \cdot)=\mathbf{S}_{-t} \mathbf{f}(t, \cdot) .
$$

Finally note that since by the very definition of $n$, see $(2.10),\|n\|_{H^{s}} \leq\|\mathbf{f}\|_{H}$, we have the following estimates for the nonlinear part,

$$
\begin{gathered}
\|\mathbf{N f}\|_{H} \lesssim\|\mathbf{f}\|_{H}^{2} . \\
\left\|\mathbf{N f}_{1}-\mathbf{N f}_{2}\right\|_{L} \lesssim\left(\left\|\mathcal{H} n_{1}\right\|_{\infty}+\left\|\mathbf{f}_{1}\right\|_{\infty}+\left\|\mathcal{H} n_{2}\right\|_{\infty}+\left\|\mathbf{f}_{2}\right\|_{\infty}\right)\left\|\mathbf{f}_{1}-\mathbf{f}_{2}\right\|_{L},
\end{gathered}
$$

which implies

$$
\left\|\mathbf{N f}_{1}-\mathbf{N f}_{2}\right\|_{L} \lesssim\left(\left\|\mathbf{f}_{1}\right\|_{H}+\left\|\mathbf{f}_{2}\right\|_{H}\right)\left\|\mathbf{f}_{1}-\mathbf{f}_{2}\right\|_{L}
$$

\section{Local in time existence}

In this section, we prove local in time existence and uniqueness for the system (2.12). The term $X$ stands for the whole space $\mathbb{R}$ or the circle $\mathbb{R} / \mathbb{Z}$. We consider the problem as a differential equation on a Banach space. Let us denote by $\mathbf{g}$ the unknown function $\mathbf{f}_{\star}$ introduced in (2.12) and rewrite it as

$$
\partial_{t} \mathbf{g}=\mathbf{S}_{-t} \mathbf{N S}_{t} \mathbf{g}
$$

The goal is to find a solution to the following fixed-point problem,

$$
\mathbf{g}(t, \cdot)=\mathbf{g}_{0}+\int_{0}^{t} \mathbf{S}_{-\tau} \mathbf{N S}_{\tau} \mathbf{g} \mathrm{d} \tau .
$$

Here the difficulty arrises from the fact that although the nonlinear term $\mathbf{N}$ may be defined on the space $L^{2}$, we need also an $L^{\infty}$ estimate in order to control the nonlinear term in $L^{2}$. Again although the Hilbert transform acts on $L^{\infty}$ functions, we need (at least) a Hölder estimate on the densities in order to control the $L^{\infty}$-norm of the Hilbert transform. Moreover the nonlinearity is not a local operator. However, the estimates given in the previous section and the appropriate properties of the space $H$ allow us to solve (3.1), despite the fact that the nonlinearity is not Lipschitz on this space. As a matter of fact, we have to show the existence of a fixed-point by an ad-hoc procedure.

To find a solution for this equation so that $\mathrm{g} \in C^{1}([0, T] ; H)$, first we choose any fixed $R>\left\|\mathbf{g}_{0}\right\|_{H}$ and then find $T$ small enough such that

$$
\left\|\mathbf{g}_{0}\right\|_{H}+C T R^{2} \leq R, \quad 2 C R T<1,
$$


where $C$ is a universal constant given by the estimates (2.14) and (2.16). We will clarify this shortly.

We construct the sequence $\left\{\mathbf{g}^{(m)}\right\}_{m}$ as follows. Let $\mathbf{g}^{(0)}(t, \cdot)=\mathbf{g}_{0}(\cdot)$ and for $m \geq 0$, define

$$
\mathbf{g}^{(m+1)}(t, \cdot)=\mathbf{g}_{0}+\int_{0}^{t} \mathbf{S}_{-\tau} \mathbf{N S}_{\tau} \mathbf{g}^{(m)}(\tau, \cdot) \mathrm{d} \tau,
$$

where the integration takes place in the space $H$. Using induction, we infer that $\mathbf{g}^{(m)} \in C^{1}([0, T] ; H)$ and that they have the uniform bound $R$, i.e. $\left\|\mathbf{g}^{(m)}\right\|_{C([0, T] ; H)} \leq R$. Now (2.16) implies

$$
\begin{aligned}
& \left\|\mathbf{g}^{(m+1)}(t, \cdot)-\mathbf{g}^{(m)}(t, \cdot)\right\|_{L} \\
& \quad \leq \int_{0}^{T}\left\|\mathbf{S}_{-\tau} \mathbf{N S}_{\tau} \mathbf{g}^{(m)}(\tau, \cdot)-\mathbf{S}_{-\tau} \mathbf{N S}_{\tau} \mathbf{g}^{(m-1)}(\tau, \cdot)\right\|_{H} \mathrm{~d} \tau \\
& \quad=\int_{0}^{T}\left\|\mathbf{N S}_{\tau} \mathbf{g}^{(m)}(\tau, \cdot)-\mathbf{N S}_{\tau} \mathbf{g}^{(m-1)}(\tau, \cdot)\right\|_{H} \mathrm{~d} \tau \\
& \quad \lesssim 2 R \int_{0}^{T}\left\|\mathbf{S}_{\tau} \mathbf{g}^{(m)}(\tau, \cdot)-\mathbf{S}_{\tau} \mathbf{g}^{(m-1)}(\tau, \cdot)\right\|_{L} \mathrm{~d} \tau \\
& \quad=2 R \int_{0}^{T}\left\|\mathbf{g}^{(m)}(\tau, \cdot)-\mathbf{g}^{(m-1)}(\tau, \cdot)\right\|_{L} \mathrm{~d} \tau .
\end{aligned}
$$

So the sequence $\left\{\mathbf{g}^{(m)}\right\}_{m}$ is Cauchy in $C([0, T] ; L)$ because of the choice of $T$. Therefore this sequence converges to a function $\mathbf{g}$ in this space.

Note that since $H^{s}$ functions are continuous (when $s>1 / 2$ ), and since for any fixed $x \in X$, the application $f \mapsto f(x)$ is an element of the dual space of $H^{s}$, hence the integral equation (3.2) is valid point-wise. We conclude that $\left\{\mathbf{g}^{(m)}\right\}_{m}$ satisfies a uniform Hölder estimate with respect to the space variable and they are uniformly Lipschitz with respect to time. Hence Arzela-Ascoli theorem implies the existence of a subsequence of $\left\{\mathbf{g}^{(m)}\right\}_{m}$ which converges uniformly on compact subsets of $[0, T] \times X$. Therefore this limit is the above mentioned function $\mathbf{g}$ and in fact there is no need to pass to a subsequence because of the uniqueness of this limit.

Now since $\mathcal{H}$ is continuous on $L^{2}$, so $\mathcal{H} \mathbf{g}^{(m)} \rightarrow \mathcal{H} \mathbf{g}$ in $L$ uniformly in time. By applying $\mathcal{H}$ to (3.2), one sees that the same uniform Hölder and Lipschitz estimates are true for $\left\{\mathcal{H} \mathbf{g}^{(m)}\right\}_{m}$. Therefore we may apply Arzela-Ascoli theorem again to the sequence $\left\{\mathcal{H} \mathbf{g}^{(m)}\right\}_{m}$ to conclude that this convergence is also uniform on compact sets and $\mathcal{H} \mathrm{g}$ is continuous and bounded.

Since $\mathbf{g}^{(m)} \in C^{0,1}([0, T] ; L)$ with uniform Lipschitz constant for all $m$, we infer that $\mathbf{g} \in C^{0,1}([0, T] ; L)$ and also we have $\mathbf{g}, \mathcal{H} n \in C_{b}([0, T] \times X)$. Therefore the mapping $\tau \mapsto \mathbf{S}_{-\tau} \mathbf{N S}_{\tau} \mathbf{g}$ is continuous with values in $L$. Hence the integration in (3.1) makes sense and the integral equation is valid as an equation in $L$ since we may pass to the limit using the estimate (2.15). Also we have $\mathbf{g} \in C^{1}([0, T] ; L)$

Fourier transform is continuous on $L^{2}$, therefore $\mathcal{F} \mathbf{g}^{(m)} \rightarrow \mathcal{F} \mathbf{g}$ in $L$ uniformly in time. We may extract a subsequence from $\left\{\mathcal{F} \mathbf{g}^{(m)}\right\}_{m}$ which converges 
almost everywhere on $X$ and Fatou's lemma implies that $\mathbf{g}(t) \in H$ for each fixed time $t$ with uniform bound for all times. Now

$$
\mathcal{F} \mathbf{g}(t, \cdot)=\mathcal{F} \mathbf{g}_{0}+\int_{0}^{t} \mathcal{F}\left(\mathbf{S}_{-\tau} \mathbf{N} \mathbf{S}_{\tau} \mathbf{g}(\tau, \cdot)\right) \mathrm{d} \tau
$$

Therefore

$$
\mathcal{F} \mathbf{g}(t+\varepsilon, \cdot)-\mathcal{F} \mathbf{g}(t, \cdot)=\int_{t}^{t+\varepsilon} \mathcal{F}\left(\mathbf{S}_{-\tau} \mathbf{N S}_{\tau} \mathbf{g}(\tau, \cdot)\right) \mathrm{d} \tau .
$$

Multiplying this equality by $\left(1+4 \pi^{2} \min \{|\xi|, \lambda\}^{2}\right)^{s / 2}$ and letting $\lambda$ to go to infinity, we conclude that $\mathbf{g} \in C([0, T] ; H)$ and hence (3.1) makes sense in the space $H$ (and in particular, the integral equality is valid everywhere). As a consequence $\mathbf{g} \in C^{1}([0, T] ; H)$ and

$$
\partial_{t} \mathbf{g}=\mathbf{S}_{-t} \mathbf{N S}_{t} \mathbf{g}
$$

as an equation in $H$. We have almost proved the following theorem.

Theorem 3.1. Suppose that the initial data $\mathbf{f}_{0}$ is in $H$. Then there exists a time $T$ such that the system (2.12) has a unique solution with properties

$$
\mathbf{f} \in L^{2}([0, T] ; H), \quad \mathbf{f}\left(0^{+}, \cdot\right)=\mathbf{f}_{0} .
$$

This solution fulfills the following properties,

$$
\mathbf{f}_{\star} \in C^{1}([0, T] ; H), \quad \mathbf{f} \in C([0, T] ; H) \cap C^{1}([0, T] ; L) .
$$

Proof. We may note that the condition $\mathbf{f} \in L^{2}([0, T] ; H)$ is sufficient to rewrite (2.12) in the integral form

$$
\mathbf{f}_{\star}(t, \cdot)=\mathbf{f}_{0}+\int_{0}^{t} \mathbf{S}_{-\tau} \mathbf{N S}_{\tau} \mathbf{f}_{\star}(\tau, \cdot) \mathrm{d} \tau,
$$

or more familiar form in the theory of semigroups,

$$
\mathbf{f}(t, \cdot)=\mathbf{S}_{t} \mathbf{f}_{0}+\int_{0}^{t} \mathbf{S}_{t-\tau} \mathbf{N} \mathbf{f}(\tau, \cdot) \mathrm{d} \tau .
$$

We have already proved the existence. Uniqueness is a consequence of estimate (2.16) and Gronwall's lemma. Clearly since $\mathbf{f}_{\star} \in C([0, T] ; H)$ and $\mathbf{f}(t, \cdot)=$ $\mathbf{S}_{t} \mathbf{f}_{\star}(t, \cdot)$, we have $\mathbf{f} \in C([0, T] ; H)$. Since $\mathbf{N}$ is continuous on $H$, therefore the integrand of the integral equation (3.4) belongs to the space $C([0, T] ; H)$ which is embedded in $C([0, T] ; L)$. Hence $\mathbf{f} \in C^{1}([0, T] ; L)$, since $\mathbf{S}_{t} \mathbf{f}_{0} \in C^{1}([0, T] ; L)$.

Theorem 3.2. Suppose that the initial data $\mathbf{f}_{0}$ is in $H$. Then there exists a maximal time $T^{*}=T^{*}\left(\mathbf{f}_{0}\right)$ such that the system $(2.12)$ has a unique solution with properties

$$
\mathbf{f} \in L_{\mathrm{loc}}^{2}\left(\left[0, T^{*}\right) ; H\right), \quad \mathbf{f}\left(0^{+}, \cdot\right)=\mathbf{f}_{0} .
$$

Also

$$
\limsup _{t \rightarrow T^{*-}}\|\mathbf{f}(t, \cdot)\|_{H}=+\infty
$$


provided that $T^{*}<+\infty$. In fact

$$
\int_{T^{*}-\varepsilon}^{T^{*}}\|\mathbf{f}(t, \cdot)\|_{H}^{2} \mathrm{~d} t=+\infty .
$$

Proof. It is easy to see that we may continue the solutions in the previous theorem after the time $T$ using $\mathbf{f}(T, \cdot)$ as the initial condition. So we may take $T^{*}$ as the supremum over all such $T$. If the last claim fails, one could use the integral equation (3.4) to define $\mathbf{f}\left(T^{*}, \cdot\right)$ because of the inequality (2.14) and continue the solution after time $T^{*}$ which is a contradiction.

Remark 3.3. We may consider this equation backward in time and the same theorem holds true for a certain time $T_{*}$ and there exists a unique solution on the interval $\left(-T_{*}, T^{*}\right)$.

Remark 3.4. One may use another approach to prove the existence of a solution. We could construct a sequence $\mathbf{f}_{m}$ whose elements are the solutions to a delayed equation as follows. For negative times define them as $\mathbf{f}_{0}$ and for $k=0, \ldots, m-1$ and $k T / m<t \leq(k+1) T / m$ let

$$
\mathbf{f}^{(m)}(t, \cdot)=\mathbf{f}^{(m)}\left(k \frac{T}{m}, \cdot\right)+\int_{k \frac{T}{m}}^{t} \mathbf{S}_{-\tau} \mathbf{N S}_{\tau} \mathbf{f}^{(m)}\left(\tau-\frac{T}{m}, \cdot\right) \mathrm{d} \tau .
$$

It is not difficult to see that using similar arguments as the proof of theorem (3.1), this sequence converges to the solution.

In the next theorem, we prove that the system (2.12) has a physical meaning in the sense that the solutions are (nonnegative) densities provided that we start the evolution from (nonnegative) densities.

Proposition 3.5. In theorem (3.2), the solutions remain nonnegative provided that the initial data are nonnegative. Also if $\mathbf{f}_{0} \in L^{1}\left(V ; L^{1}\right)$ then the solution remains in $L^{1}$ (This is automatically the case for the circle) and the total mass of the solution is conserved.

Proof. Since $\|\mathcal{H} n\|_{\infty}$ is controlled by a constant $C(T)$ on $[0, T]$ where $T<T^{*}$, looking at the equation (3.3), we may conclude that for $0 \leq t_{1}<t_{2} \leq T$, (recall that $\mathbf{f}(\tau, \cdot)=\mathbf{S}_{\tau} \mathbf{f}_{\star}(\tau, \cdot)$ )

$$
\begin{aligned}
& \mathbf{f}_{\star v}\left(t_{2}, x\right)-\mathbf{f}_{\star v}\left(t_{1}, x\right) \\
& \quad=\int_{t_{1}}^{t_{2}} \mathbf{S}_{-\tau} \mathbf{N} \mathbf{f}(\tau, x) \mathrm{d} \tau \\
& =\int_{t_{1}}^{t_{2}}\left([v \mathcal{H} n]_{-} n-\left(\mu_{+}[\mathcal{H} n]_{-}+\mu_{-}[\mathcal{H} n]_{+}\right) \mathbf{f}_{v}\right)(\tau, x+v \tau) \mathrm{d} \tau \\
& \quad \geq-C(T) \int_{t_{1}}^{t_{2}}\left(|v|[n]_{-}+\mu[\mathbf{f}]_{+}\right)(\tau, x+v \tau) \mathrm{d} \tau \\
& \quad=-C(T) \int_{t_{1}}^{t_{2}}\left(|v|\left[\sum_{v^{\prime} \in V} \mathbf{f}_{\star v^{\prime}}\left(\tau, x-v^{\prime} \tau+v \tau\right)\right]_{-}+\mu\left[\mathbf{f}_{\star v}(\tau, x)\right]_{+}\right) \mathrm{d} \tau,
\end{aligned}
$$


where $\mu=\mu_{+}+\mu_{-}=\sum_{v \in V}|v|$ and by $\mathbf{f}_{\star v}$ we mean the $v$-th component of the vector $\mathbf{f}_{\star}$. Fix $x$ and $v$. We claim that

$$
\mathbf{f}_{\star v}(t, x) \geq-C(T) \int_{0}^{t}|v|\left[\sum_{v^{\prime} \in V} \mathbf{f}_{\star v^{\prime}}\left(\tau, x-v^{\prime} \tau+v \tau\right)\right]_{-} \mathrm{d} \tau .
$$

Assume by the way of contradiction that this is not the case at an instant $t_{2} \leq T$. So $\mathbf{f}_{\star v}\left(t_{2}, x\right)<0$ and since $\mathbf{f}_{\star v}(\cdot, x)$ is a continuous function of time and it is nonnegative for $t=0$, hence there exists a time $t_{1} \geq 0$ such that $\mathbf{f}_{\star v}(\cdot, x)$ is negative on the time interval $\left(t_{1}, t_{2}\right)$ and $\mathbf{f}_{\star v}\left(t_{1}, x\right)=0$. Now $(3.5)$ implies

$$
\begin{aligned}
\mathbf{f}_{\star v}\left(t_{2}, x\right) & \geq-C(T) \int_{t_{1}}^{t_{2}}|v|\left[\sum_{v^{\prime} \in V} \mathbf{f}_{\star v^{\prime}}\left(\tau, x-v^{\prime} \tau+v \tau\right)\right]_{-} \mathrm{d} \tau \\
& \geq-C(T) \int_{0}^{t_{2}}|v|\left[\sum_{v^{\prime} \in V} \mathbf{f}_{\star v^{\prime}}\left(\tau, x-v^{\prime} \tau+v \tau\right)\right]_{-} \mathrm{d} \tau,
\end{aligned}
$$

which is a contradiction. Now define

$$
m_{v}(t)=\inf _{x \in X} \mathbf{f}_{\star v}(t, x), \quad m(t)=\sum_{v \in V} m_{v}(t) .
$$

Note that $a \geq b$ implies $[a]_{-} \leq[b]_{-}$and therefore

$$
\begin{aligned}
-\mathbf{f}_{\star v}(t, x) & \leq C(T) \int_{0}^{t}|v|\left[\sum_{v^{\prime} \in V} \mathbf{f}_{\star v^{\prime}}\left(\tau, x-v^{\prime} \tau+v \tau\right)\right]_{-} \mathrm{d} \tau \\
& \leq C(T)|v| \int_{0}^{t}[m(\tau)]_{-} \mathrm{d} \tau .
\end{aligned}
$$

Taking the supremum over $x$, we conclude that

$$
-m_{v}(t) \leq C(T)|v| \int_{0}^{t}[m(\tau)]_{-} \mathrm{d} \tau
$$

Taking the sum over $v$ implies

$$
[m(t)]_{-}=\max \{-m(t), 0\} \leq C(T) \mu \int_{0}^{t}[m(\tau)]_{-} \mathrm{d} \tau .
$$

Applying Gronwall's lemma implies that $[m(t)]_{-}=0$ since $[m(0)]_{-}=0$ and (3.7) completes the proof.

For the last part, note that $\mathbf{N g}$ is in $L^{1}\left(V ; L^{1}(\mathbb{R})\right)$ provided that $\mathbf{g} \in$ $L=L^{1}\left(V ; L^{2}(\mathbb{R})\right)$ and since $\mathbf{f}(t, \cdot)$ is bounded in $L$, the $L^{1}$-norm of $\mathbf{N f}$ is uniformly bounded in time. Integrating the components of both sides of (3.4) 
with respect to $x$ and using Fubini's theorem yields

$$
\begin{aligned}
\int_{X} \mathbf{f}_{v}(t, x) \mathrm{d} x & =\int_{X} \mathcal{S}_{v t} \mathbf{f}_{0 v}(x) \mathrm{d} x+\int_{X} \int_{0}^{t} \mathcal{S}_{v(t-\tau)}(\mathbf{N f})_{v}(\tau, x) \mathrm{d} \tau \mathrm{d} x \\
& =\int_{X} \mathbf{f}_{0 v}(x-v t) \mathrm{d} x+\int_{0}^{t} \int_{X} \mathcal{S}_{v(t-\tau)}(\mathbf{N f})_{v}(\tau, x) \mathrm{d} x \mathrm{~d} \tau \\
& =\int_{X} \mathbf{f}_{0 v}(x) \mathrm{d} x+\int_{0}^{t} \int_{X}(\mathbf{N f})_{v}(\tau, x) \mathrm{d} x \mathrm{~d} \tau .
\end{aligned}
$$

Taking the sum over $v$ implies that

$$
\int_{X} n(t, x) \mathrm{d} x=\int_{X} n_{0}(x) \mathrm{d} x,
$$

since $\sum_{v \in V}(\mathbf{N g})_{v}=0$ for every $\mathbf{g} \in L$.

Remark 3.6. Observe that this existence result does not use the full nature of the Hilbert transform. In fact it is enough to have a bounded operator, e.g. if we take any (singular) convolution operator which is bounded on $H^{s}$. This is the case for the generalized problem in which $\alpha>0$. Also note that this method does not work in higher dimensions. Here we need to embed the space $H^{s}$ into $L^{\infty}$ which pushes us to choose $s>d / 2$ and on the other hand the truncation operator limits us to the case $s \leq 1$.

Remark 3.7. It is not difficult to see that we may pass to the limit to solve the problem in the case where the set of admissible velocities $V$ is an interval (or more generally any bounded measurable set).

We continue by establishing some estimates to find a lower bound for the lifetime of the solution in the case $s=1$ and $V=\{+1,-1\}$; the most simple and interesting case. We denote by $p$ and $q$ the components of $\mathbf{f}$. So the problem reads as follows

$$
\left\{\begin{array}{l}
\partial_{t} p+\partial_{x} p=-[\mathcal{H} n]_{+} p+[\mathcal{H} n]_{-} q, \\
\partial_{t} q-\partial_{x} q=[\mathcal{H} n]_{+} p-[\mathcal{H} n]_{-} q
\end{array}\right.
$$

Proposition 3.8. If the initial data is symmetric in the sense that $p_{0}(x)=$ $q_{0}(-x)$ for $x \in X$, then the solution remains symmetric.

Proof. It is easy to check that if the pair $(p(t, x), q(t, x))$ is a solution to (3.8), so be $(q(t,-x), p(t,-x))$, and the result follows using the uniqueness of the solution.

Let us start from

$$
\partial_{t} p_{\star}=\mathcal{S}_{-t} N\left(\mathcal{S}_{t} p_{\star}, \mathcal{S}_{-t} q_{\star}\right)=\mathcal{S}_{-t} N(p, q),
$$

where $p_{\star}=\mathcal{S}_{-t} p$ and $q_{\star}=\mathcal{S}_{t} q$ and $N(f, g)=-[\mathcal{H}(f+g)]_{+} f+[\mathcal{H}(f+g)]_{-} g$. Consider the bilinear form $\mathcal{D}(f, g)=\int \partial_{x} f \partial_{x} g$ and since $p_{\star}, q_{\star} \in C^{1}\left([0, T] ; H^{1}\right)$, it is clear that

$$
\frac{\mathrm{d}}{\mathrm{d} t} \mathcal{D}\left(p_{\star}, p_{\star}\right)=2 \mathcal{D}\left(p_{\star}, \partial_{t} p_{\star}\right)=-2 \mathcal{D}\left(p_{\star}, \mathcal{S}_{-t} N(p, q)\right) .
$$


But $\mathcal{D}$ is invariant under translations, so

$$
\frac{\mathrm{d}}{\mathrm{d} t} \mathcal{D}(p, p)=-2 \mathcal{D}(p, \mathbf{N}(p, q)) .
$$

Therefore

$$
\begin{aligned}
\frac{1}{2} & \frac{\mathrm{d}}{\mathrm{d} t} \int\left|\partial_{x} p\right|^{2} \\
& =\int \partial_{x} p \partial_{x}\left([\mathcal{H} n]_{-} q-[\mathcal{H} n]_{+} p\right) \\
& =-\int[\mathcal{H} n]_{+}\left|\partial_{x} p\right|^{2}+\int[\mathcal{H} n]_{-} \partial_{x} p \partial_{x} q-\int \partial_{x} p\left(p \partial_{x}[\mathcal{H} n]_{+}-q \partial_{x}[\mathcal{H} n]_{-}\right)
\end{aligned}
$$

Similarly,

$$
\begin{aligned}
\frac{1}{2} & \frac{\mathrm{d}}{\mathrm{d} t} \int\left|\partial_{x} q\right|^{2} \\
\quad & =-\int[\mathcal{H} n]_{-}\left|\partial_{x} q\right|^{2}+\int[\mathcal{H} n]_{+} \partial_{x} p \partial_{x} q+\int \partial_{x} q\left(p \partial_{x}[\mathcal{H} n]_{+}-q \partial_{x}[\mathcal{H} n]_{-}\right) .
\end{aligned}
$$

Hence

$$
\begin{aligned}
\frac{1}{2} \frac{\mathrm{d}}{\mathrm{d} t} & \left\|\left(\partial_{x} p, \partial_{x} q\right)\right\|_{2}^{2} \\
= & \int[\mathcal{H} n]_{+} \partial_{x} p\left(\partial_{x} q-\partial_{x} p\right)+\int[\mathcal{H} n]_{-} \partial_{x} q\left(\partial_{x} q-\partial_{x} p\right) \\
& +\int\left(\partial_{x} q-\partial_{x} p\right)\left(p \partial_{x}[\mathcal{H} n]_{+}-q \partial_{x}[\mathcal{H} n]_{-}\right) \\
\leq & \frac{1}{4}\left\|\left(\partial_{x} q, \partial_{x} p\right)\right\|_{2}^{2}\|\mathcal{H} n\|_{\infty}+\left\|\partial_{x} q-\partial_{x} p\right\|_{2}\left\|\partial_{x} \mathcal{H} n\right\|_{2}\|(p, q)\|_{\infty}
\end{aligned}
$$

since for any real numbers $a, b$, we have $a(b-a) \leq b^{2} / 4$. Now we compute

$$
\begin{aligned}
\left\|\partial_{x} q-\partial_{x} p\right\|_{2}\left\|\partial_{x} \mathcal{H} n\right\|_{2} & \leq\left\|\partial_{x} q-\partial_{x} p\right\|_{2}\left\|\partial_{x} n\right\|_{2} \\
& \leq \frac{1}{2}\left(\left\|\partial_{x} q-\partial_{x} p\right\|_{2}^{2}+\left\|\partial_{x} p+\partial_{x} q\right\|_{2}^{2}\right) \\
& =\left\|\left(\partial_{x} p, \partial_{x} q\right)\right\|_{2}^{2},
\end{aligned}
$$

which implies

$$
\frac{\mathrm{d}}{\mathrm{d} t}\left\|\left(\partial_{x} p, \partial_{x} q\right)\right\|_{2} \leq\left(\frac{1}{4}\|\mathcal{H} n\|_{\infty}+\|(p, q)\|_{\infty}\right)\left\|\left(\partial_{x} p, \partial_{x} q\right)\right\|_{2} .
$$

This inequality is enough to find a control on $\left\|\left(\partial_{x} p, \partial_{x} q\right)\right\|_{2}$ using Nash inequality (2.4). Also one may use (2.5) on the circle. However, we may provide slightly better results if we use the (more accurate) estimate (2.3). To do this, we need an estimate on the $L^{2}$ norms of $p$ and $q$. This can be done using similar argument as the proof of (3.9), and we conclude that

$$
\frac{\mathrm{d}}{\mathrm{d} t}\|(p, q)\|_{2} \leq \frac{1}{4}\|\mathcal{H} n\|_{\infty}\|(p, q)\|_{2} .
$$

So it is reasonable to define

$$
W=\left(\left\|\left(\partial_{x} p, \partial_{x} q\right)\right\|_{2}\|(p, q)\|_{2}\right)^{1 / 2},
$$


and we have

$$
\frac{\mathrm{d}}{\mathrm{d} t} W \leq\left(\frac{1}{4}\|\mathcal{H} n\|_{\infty}+\frac{1}{2}\|(p, q)\|_{\infty}\right) W .
$$

We may use lemma (2.3) to find the following estimates,

$$
\begin{aligned}
\|\mathcal{H} n\|_{\infty} & \leq\left(\|p+q\|_{2}\left\|\partial_{x} p+\partial_{x} q\right\|_{2}\right)^{1 / 2} \leq \sqrt{2} W \\
\|(p, q)\|_{\infty} & \leq\left(\|(p, q)\|_{2}\left\|\left(\partial_{x} p, \partial_{x} q\right)\right\|_{2}\right)^{1 / 2}=W, \quad(X=\mathbb{R}) .
\end{aligned}
$$

If we are on the circle, we must add $M$ to the right hand side of the last inequality. In the case of the symmetric initial data, we have

$$
\|(p, q)\|_{\infty} \leq\left(\frac{1}{2}\|(p, q)\|_{2}\left\|\left(\partial_{x} p, \partial_{x} q\right)\right\|_{2}\right)^{1 / 2}=\frac{1}{\sqrt{2}} W .
$$

Again if we are on the circle, we must add $M / 2$ to the r.h.s. of this inequality. We summarize,

where

$$
\begin{array}{lrl}
\frac{\mathrm{d}}{\mathrm{d} t} W & \leq \gamma W^{2}, & (X=\mathbb{R}), \\
\frac{\mathrm{d}}{\mathrm{d} t} W \leq(m+\gamma W) W, & (X=\mathbb{R} / \mathbb{Z}),
\end{array}
$$

$$
\begin{aligned}
\gamma=\frac{2+\sqrt{2}}{4}, & \gamma_{\mathrm{sym}} & =\frac{1}{\sqrt{2}}, \\
m=\frac{M}{2}, & m_{\mathrm{sym}} & =\frac{M}{4} .
\end{aligned}
$$

Now we are able to state a lower bound for the lifetime of the solutions.

Theorem 3.9. In theorem (3.2), we have

$$
\begin{array}{ll}
T^{*}\left(p_{0}, q_{0}\right) \geq \frac{1}{\gamma\left(\left\|\left(p_{0}, q_{0}\right)\right\|_{2}\left\|\left(\partial_{x} p_{0}, \partial_{x} q_{0}\right)\right\|_{2}\right)^{1 / 2}}, & (X=\mathbb{R}), \\
T^{*}\left(p_{0}, q_{0}\right) \geq \frac{1}{m} \log \left(1+\frac{m}{\gamma\left(\left\|\left(p_{0}, q_{0}\right)\right\|_{2}\left\|\left(\partial_{x} p_{0}, \partial_{x} q_{0}\right)\right\|_{2}\right)^{1 / 2}}\right), & (X=\mathbb{R} / \mathbb{Z}),
\end{array}
$$

and $m, \gamma$ is defined as (3.12) in the general or symmetric case.

Proof. In the case $X=\mathbb{R}$ from the estimate (3.11) for $\frac{\mathrm{d}}{\mathrm{d} t} W$, one has

$$
W(t) \leq \frac{W_{0}}{1-\gamma W_{0} t}
$$

which implies using (3.9),

$$
\frac{\mathrm{d}}{\mathrm{d} t}\left\|\left(\partial_{x} p, \partial_{x} q\right)\right\|_{2} \leq C W\left\|\left(\partial_{x} p, \partial_{x} q\right)\right\|_{2} \leq \frac{C W_{0}}{1-\gamma W_{0} t}\left\|\left(\partial_{x} p, \partial_{x} q\right)\right\|_{2} .
$$

Hence

$$
\left\|\partial_{x} p, \partial_{x} q\right\|_{2} \leq \frac{\left\|\left(\partial_{x} p_{0}, \partial_{x} q_{0}\right)\right\|_{2}}{\left(1-\gamma W_{0} t\right)^{C / \gamma}}
$$

Similarly we may conclude that $\|(p, q)\|_{2}$ is also bounded until time $1 /\left(\gamma W_{0}\right)$ and the result follows using theorem (3.2). 
In the case $X=\mathbb{R} / \mathbb{Z}$, from (3.11) we have,

$$
W(t) \leq \frac{m \gamma^{-1}}{\left(1+m \gamma^{-1} W_{0}^{-1}\right) \mathrm{e}^{-m t}-1} .
$$

Since

$$
\left\|\left(\partial_{x} p, \partial_{x} q\right)\right\|_{2}=\|(p, q)\|_{2}^{-1} W^{2} \leq \frac{1}{\sqrt{2} M} W^{2},
$$

again theorem (3.2) completes the proof.

Remark 3.10. As we mentioned earlier, on the circle, we may use lemma (2.6),

$$
\begin{aligned}
\|\mathcal{H} n\|_{\infty} & \leq \frac{1}{2 \sqrt{3}}\left\|\partial_{x} n\right\|_{2} \leq \frac{1}{\sqrt{6}}\left\|\left(\partial_{x} p, \partial_{x} q\right)\right\|_{2}, \\
\|(p, q)\|_{\infty} & \leq M+\frac{1}{2 \sqrt{3}}\left\|\left(\partial_{x} p, \partial_{x} q\right)\right\|_{2} .
\end{aligned}
$$

In the case of the symmetric initial data, we may improve the second one,

$$
\|(p, q)\|_{\infty} \leq \frac{M}{2}+\frac{1}{2 \sqrt{6}}\left\|\left(\partial_{x} p, \partial_{x} q\right)\right\|_{2} .
$$

Therefore inequality (3.9) implies

$$
\frac{\mathrm{d}}{\mathrm{d} t}\left\|\left(\partial_{x} p, \partial_{x} q\right)\right\|_{2} \leq\left(2 m+\beta\left\|\left(\partial_{x} p, \partial_{x} q\right)\right\|_{2}\right)\left\|\left(\partial_{x} p, \partial_{x} q\right)\right\|_{2},
$$

where $m$ is defined in (3.12) and

$$
\beta=\frac{1}{2 \sqrt{3}}+\frac{1}{4 \sqrt{6}}, \quad \beta_{\mathrm{sym}}=\frac{\sqrt{3}}{4 \sqrt{2}} .
$$

We conclude that

$$
T^{*}\left(p_{0}, q_{0}\right) \geq \frac{1}{2 m} \log \left(1+\frac{2 m}{\beta\left\|\left(\partial_{x} p_{0}, \partial_{x} q_{0}\right)\right\|_{2}}\right), \quad(X=\mathbb{R} / \mathbb{Z}) .
$$

\section{Numerical schemes and discrete hilbert transform}

In this section, we present a numerical simulation for this system, as well as the proof of the convergence for the numerical scheme which is used here. Here we introduce a method which is very easy to implement and relies on a method which may be labeled a "method of lines". We shall be more specific in a moment. First we reduce the problem to a family of finite dimensional subspaces of $H$ and prove that these solutions will converge to the original one provided that some natural hypotheses are satisfied.

Theorem 4.1. Assume that $\left\{E_{k}\right\}_{k}$ is a sequence of finite dimensional subspaces of $H^{s}(X)$ which are invariant under the action of the shift operator. Let $H_{k}=$ $L^{1}\left(V ; E_{k}\right) \hookrightarrow H$ and suppose that the projection mappings $\left\{\mathbb{P}_{k}: H \rightarrow H_{k}\right\}_{k}$ satisfy the uniform estimate

$$
\left\|\mathbb{P}_{k} \mathbf{g}-\mathbf{g}\right\|_{L} \lesssim \omega_{k}\left\|\mathbb{P}_{k} \mathbf{g}-\mathbf{g}\right\|_{H},
$$


where $\left\{\omega_{k}\right\}$ is a bounded sequence. Also assume that for each fixed $\mathbf{g} \in H$, we have,

$$
\left\|\mathbb{P}_{k} \mathbf{g}-\mathbf{g}\right\|_{H} \rightarrow 0 .
$$

(The convergence is not necessarily uniform on $\mathbf{g}$ ). Then there exists a positive time $T$ such that the system

$$
\mathbf{S}_{t} \partial_{t} \mathbf{S}_{-t} \mathbf{f}^{(k)}=\mathbb{P}_{k} \mathbf{N} \mathbf{f}^{(k)},
$$

with the initial data $\mathbb{P}_{k} \mathbf{f}_{0}$, has a unique solution in $H_{k}$ on the time interval $[0, T]$ and

$$
\sup _{0 \leq t \leq T}\left\|\mathbf{f}^{(k)}-\mathbf{f}\right\|_{L}=o\left(\omega_{k}\right),
$$

where $\mathbf{f}$ solves the system (2.12). In fact $\mathbf{f}^{(k)}$ converges also uniformly on bounded sets of $[0, T] \times X$ to $\mathbf{f}$. Moreover in the case $s=1$, if $\omega_{k}$ converges to zero,

$$
\sup _{0 \leq t \leq T}\left\|\Delta_{w_{k}} \mathbf{f}^{(k)}-\partial_{x} \mathbf{f}\right\|_{L}=o(1),
$$

where $\Delta_{h}$ denotes the discrete differentiation, i.e.

$$
\Delta_{h} u(x)=\frac{u(x+h)-u(x)}{h} .
$$

Proof. From (4.2), using BanachSteinhaus theorem, we conclude that

$$
\left\|\mathbb{P}_{k} \mathbf{g}\right\|_{H} \lesssim\|\mathbf{g}\|_{H}
$$

uniformly in $k$ and $\mathbf{g}$. Since $E_{k}$ is finite dimensional, the $L$-norm and $H$-norm are equivalent on it and hence the nonlinear part is Lipschitz on bounded sets of $H_{k}$ because of (4.4) and (2.16). Therefore (4.3) has a unique solution and because of the uniform estimate (2.14) and boundedness of $\mathbb{P}_{k} \mathbf{f}_{0}$, there exists a positive time $T$ such that we have a solution in the time interval $[0, T]$ for all $k$. More precisely, since $\mathbf{f}_{\star}^{(k)} \in C^{1}([0, t], H)$, from

$$
\partial_{t} \mathbf{f}_{\star}^{(k)}=\mathbf{S}_{-t} \mathbb{P}_{k} \mathbf{N S}_{t} \mathbf{f}_{\star}^{(k)},
$$

we have

$$
\begin{aligned}
\partial_{t}\left\|\mathbf{f}_{\star}^{(k)}\right\|_{H} & \leq\left\|\partial_{t} \mathbf{f}_{\star}^{(k)}\right\|_{H} \\
& =\left\|\mathbb{P}_{k} \mathbf{N S}_{t} \mathbf{f}_{\star}^{(k)}\right\|_{H} \\
& \leq C\left\|\mathbf{f}_{\star}^{(k)}\right\|_{H}^{2},
\end{aligned}
$$

which implies

$$
\begin{aligned}
\left\|\mathbf{f}^{(k)}\right\|_{H} & =\left\|\mathbf{f}_{\star}^{(k)}\right\|_{H} \\
& \leq \frac{\left\|\mathbb{P}_{k} \mathbf{f}_{0}\right\|_{H}}{1-C\left\|\mathbb{P}_{k} \mathbf{f}_{0}\right\|_{H} t} \\
& \leq \frac{C\left\|\mathbf{f}_{0}\right\|_{H}}{1-C\left\|\mathbf{f}_{0}\right\|_{H} t} .
\end{aligned}
$$


Using theorem (3.2), we conclude that we may choose $T \lesssim\left\|\mathbf{f}_{0}\right\|_{H}^{-1}$. Also all these solutions have a uniform bound in $H$ and looking at the integral form of the equations

$$
\begin{aligned}
\mathbf{f}^{(k)}(t, \cdot) & =\mathbf{S}_{t} \mathbf{f}_{0}^{(k)}+\int_{0}^{t} \mathbf{S}_{t-\tau} \mathbb{P}_{k} \mathbf{N} \mathbf{f}^{(k)}(\tau, \cdot) \mathrm{d} \tau \\
\mathbf{f}(t, \cdot) & =\mathbf{S}_{t} \mathbf{f}_{0}+\int_{0}^{t} \mathbf{S}_{t-\tau} \mathbf{N f}(\tau, \cdot) \mathrm{d} \tau
\end{aligned}
$$

yields

$$
\begin{aligned}
& \left\|\mathbf{f}^{(k)}-\mathbf{f}\right\|_{L} \\
& \quad \leq\left\|\mathbb{P}_{k} \mathbf{f}_{0}-\mathbf{f}_{0}\right\|_{L}+\int_{0}^{t}\left\|\mathbb{P}_{k} \mathbf{N} \mathbf{f}^{(k)}-\mathbf{N f}\right\|_{L} \mathrm{~d} \tau \\
& \quad \leq\left\|\mathbb{P}_{k} \mathbf{f}_{0}-\mathbf{f}_{0}\right\|_{L}+\int_{0}^{t}\left\|\mathbb{P}_{k} \mathbf{N} \mathbf{f}^{(k)}-\mathbf{N} \mathbf{f}^{(k)}\right\|_{L} \mathrm{~d} \tau+\int_{0}^{t}\left\|\mathbf{N} \mathbf{f}^{(k)}-\mathbf{N} \mathbf{f}\right\|_{L} \mathrm{~d} \tau \\
& \quad \leq\left\|\mathbb{P}_{k} \mathbf{f}_{0}-\mathbf{f}_{0}\right\|_{L}+\int_{0}^{T}\left\|\mathbb{P}_{k} \mathbf{N} \mathbf{f}^{(k)}-\mathbf{N} \mathbf{f}^{(k)}\right\|_{L} \mathrm{~d} \tau+C \int_{0}^{t}\left\|\mathbf{f}^{(k)}-\mathbf{f}\right\|_{L} \mathrm{~d} \tau
\end{aligned}
$$

where the constant $C$ depends only on the initial data. Gronwall's lemma implies

$$
\begin{aligned}
\left\|\mathbf{f}^{(k)}-\mathbf{f}\right\|_{L} & \leq \mathrm{e}^{C t}\left(\left\|\mathbb{P}_{k} \mathbf{f}_{0}-\mathbf{f}_{0}\right\|_{L}+\int_{0}^{T}\left\|\mathbb{P}_{k} \mathbf{N} \mathbf{f}^{(k)}-\mathbf{N} \mathbf{f}^{(k)}\right\|_{L} \mathrm{~d} \tau\right) \\
& \lesssim \omega_{k}\left(\left\|\mathbb{P}_{k} \mathbf{f}_{0}-\mathbf{f}_{0}\right\|_{H}+\int_{0}^{T}\left\|\mathbb{P}_{k} \mathbf{N} \mathbf{f}^{(k)}-\mathbf{N f}^{(k)}\right\|_{H} \mathrm{~d} \tau\right)
\end{aligned}
$$

Now from (4.2) together with Lesbesque's dominated convergence theorem, we infer that $\left\|\mathbf{f}^{(k)}-\mathbf{f}\right\|_{L}=o\left(\omega_{k}\right)$. Therefore $\mathbf{f}^{(k)}$ converges to $\mathbf{f}$ in $L$ uniformly in time. Also the sequence $\left\{\mathbf{f}^{(k)}\right\}_{k}$ satisfies a uniform Hölder estimate with respect to space variable and a uniform Lipschitz estimate with respect to time. Hence applying Arzela-Ascoli theorem to this sequence implies that there exists a subsequence of $\left\{\mathbf{f}^{(k)}\right\}_{k}$ which converges uniformly on bounded sets and because of the $L$-convergence, we infer that this limit is unique and equals to $\mathbf{f}$ and we do not need to pass to a subsequence.

For the last part, since $\left\|\Delta_{h} f\right\|_{2} \leq 2\|f\|_{2} / h$ for every $f \in L^{2}$, we have

$$
\left\|\Delta_{\omega_{k}} \mathbf{f}^{(k)}-\Delta_{\omega_{k}} \mathbf{f}\right\|_{L}=o(1) .
$$

Also $\left\|\Delta_{\omega_{k}} f-\partial_{x} f\right\|_{2}=o(1)$ for $f \in H^{1}$ and since $\mathbf{f} \in C([0, T] ; H)$ and $[0, T]$ is compact, this convergence is uniform in time for $\mathbf{f}$ and the proof is complete.

Remark 4.2. Note that in fact the convergence is true as long as the solution exists in the time interval $[0, T]$. The uniform estimate (4.4) ensures us that such time interval exists.

In the following we assume that we are in the case of the circle, i.e. $X=\mathbb{R} / \mathbb{Z}$. The case of an initial data with compact support in the whole 
space has no major difference. Indeed for $t<T$, the function $\mathbf{f}(t, \cdot)$ has its support included in $\left[-L-v_{\max } t, L+v_{\max } t\right]$ where $\mathbf{f}_{0}$ is supported in $[-L, L]$ and $v_{\max }=\max \{|v|\}$.

Once we know the above convergence result, we may solve this problem in the finite dimensional spaces $E_{k}$ using the standard numerical methods for ODE's, e.g. the standard Runge-Kutta method. A simple way is to choose the spaces $E_{k}$ to be the span of the functions $\mathrm{e}^{2 \pi \mathrm{i} \xi x}$ for $|\xi| \leq k$ and to use the discrete Fourier transform to calculate the Hilbert transform or its generalized version (i.e. when $\alpha>0$ ). The advantage is that we may also use the point-wise multiplication and truncation.

We investigate here how an efficient discrete Hilbert transform can be defined using discrete Fourier transform. For a vector $b=\left(b_{-k}, \ldots, b_{k}\right)$ of complex numbers, the "discrete Fourier transform" (DFT in short) of this vector, i.e. $\beta=\mathcal{F}_{\mathrm{d}} b$, is defined as

$$
\beta_{j}=\left(\mathcal{F}_{\mathrm{d}} b\right)_{j}=\frac{1}{2 k+1} \sum_{|m| \leq k} b_{m} \mathrm{e}^{-2 \pi \mathrm{i} m j /(2 k+1)}, \quad|j| \leq k .
$$

It is easy to see that the inverse of DFT is

$$
b_{m}=\left(\mathcal{F}_{\mathrm{d}}^{-1} \beta\right)_{m}=\sum_{|j| \leq k} \beta_{j} \mathrm{e}^{2 \pi \mathrm{i} j m /(2 k+1)}, \quad|m| \leq k .
$$

In fact we may look at the vector $b$ as the representation of the function

$$
\widetilde{b}(x)=\sum_{|j| \leq k} \beta_{j} \mathrm{e}^{2 \pi \mathrm{i} j x},
$$

which belongs to $E_{k}$. In this point of view, $b$ is the $(2 k+1)$-point sampling of the function $\widetilde{b}$ at the points $x_{m}:=m h$ for $|m| \leq k$, where

$$
h=(2 k+1)^{-1}
$$

is the space variable step, and

$$
\widetilde{\mathcal{F}}(j)=\left(\mathcal{F}_{\mathrm{d}} b\right)_{j}, \quad|j| \leq k .
$$

$E_{k}$ is invariant under translations and if we define the discrete shift operator as

$$
\mathcal{S}_{y} b=\mathcal{F}_{\mathrm{d}}^{-1}\left(\mathrm{e}^{-2 \pi \mathrm{i} j y} \mathcal{F}_{\mathrm{d}} b\right)
$$

it agrees with the shift operator (2.14) in the sense that $\widetilde{\mathcal{S}_{y} b}=\mathcal{S}_{y} \widetilde{b}$. Also it is clear that if $z$ is an integer number, then $\left(\mathcal{S}_{z h} b\right)_{m}=b_{m-z}$ where $m-z$ is calculated modulus $2 k+1$.

Also $E_{k}$ is invariant under the generalized Hilbert transform. We may define the generalized discrete Hilbert transform of a vector $b$ using its DFT by

$$
\mathcal{H} b=\mathcal{F}_{\mathrm{d}}^{-1}\left(\frac{-2 \pi j}{\alpha+2 \pi|j|} \mathcal{F}_{\mathrm{d}} b\right)
$$


and this definition coincides with the usual definition $(2.1)$, i.e. $\widetilde{\mathcal{H} b}=\mathcal{H} \widetilde{b}$. In fact in the case of $\alpha=0$, we can do straightforward calculations to obtain

$$
\mathbb{H} \widetilde{b}(x)=\sum_{|m| \leq k} b_{m} \Lambda_{h}\left(x-x_{m}\right), \quad \Lambda_{h}(x)=\frac{\cos (\pi x)-\cos \left(h^{-1} \pi x\right)}{\sin (\pi x)}
$$

Therefore the very definition of discrete Hilbert transform is the most natural choice in another point of view. Noting that $\cot \pi x$ is the kernel of the Hilbert transform on the circle, in order to calculate the numerical convolution of $\widetilde{b}$ with this kernel at the points $x_{m}$, we begin with the calculation of that convolution at the points $x_{m+1 / 2}$, so that the singularity of the kernel at zero is avoided, then we construct an element of $E_{k}$ which takes these calculated values at $x_{m+1 / 2}$, and finally we evaluate this element of $E_{k}$ at the points $x_{m}$.

It only remains to analyze the truncation and multiplication operators. The simplest and natural choice is to use the samples for the calculation of each discretization. This corresponds to the natural projection of functions of $H^{1}$ onto the space $E_{k}$ by using their samples at the points $x_{m}$. More precisely, fix $k$ and for $f \in H^{1}$, denote $b(f)_{m}:=f\left(x_{m}\right)$ for $|m| \leq k$ and define the linear projection map $\mathbb{P}_{k}: H^{1} \rightarrow E_{k}$ as being $\mathbb{P}_{k} f=\widetilde{b(f)}$. Recalling the definition of DFT, we have

$$
\mathbb{P}_{k} f(x)=h \sum_{|m| \leq k} f\left(x_{m}\right) K_{h}\left(x-x_{m}\right)
$$

where $K_{h}$ is the so-called Dirichlet kernel,

$$
K_{h}(x)=\sum_{|j| \leq k} \mathrm{e}^{2 \pi \mathrm{i} j x}=\frac{\sin \left(h^{-1} \pi x\right)}{\sin (\pi x)}
$$

In fact $\mathbb{P}_{k} f$ is the interpolation of the function $f$, by means of elements of the space $E_{k}$, at the collocation points $x_{m}$. The integral of $K_{h}$ is equal to 1 , therefore

$$
\int_{X} \mathbb{P}_{k} f(x) \mathrm{d} x=h \sum_{|m| \leq k} f\left(x_{m}\right)
$$

Moreover, it is clear that $\left\{K_{h}\left(x-x_{m}\right)\right\}_{|m| \leq k}$ is a set of perpendicular functions in $L^{2}$ with the norm $h^{-1 / 2}$. Hence

$$
\left\|\mathbb{P}_{k} f\right\|_{2}^{2}=h \sum_{|m| \leq k}\left|f\left(x_{m}\right)\right|^{2}
$$

Although $\mathbb{P}_{k}$ does not commute with the shift operator in general, it commutes with $\mathcal{S}_{h}$. We conclude that 


$$
\begin{aligned}
\left\|\Delta_{h} \mathbb{P}_{k} f\right\|_{2}^{2}=\left\|\mathbb{P}_{k} \Delta_{h} f\right\|_{2}^{2} & =h \sum_{|m| \leq k}\left(\frac{f\left(x_{m+1}\right)-f\left(x_{m}\right)}{h}\right)^{2} \\
& =h^{-1} \sum_{|m| \leq k}\left(\int_{x_{m}}^{x_{m+1}} \partial_{x} f\right)^{2} \\
& \leq \sum_{|m| \leq k} \int_{x_{m}}^{x_{m+1}}\left|\partial_{x} f\right|^{2} \\
& =\left\|\partial_{x} f\right\|_{2}^{2} .
\end{aligned}
$$

On the other hand, if we represent $\mathbb{P}_{k} f=\sum_{|j| \leq k} \beta(f)_{j} \mathrm{e}^{2 \pi \mathrm{i} j x} \in E_{k}$,

$$
\begin{aligned}
\left\|\partial_{x} \mathbb{P}_{k} f\right\|_{2}^{2} & =\sum_{|j| \leq k} 4 \pi^{2} j^{2}\left|\beta(f)_{j}\right|^{2} \\
& \leq\left(\sup _{|j| \leq k} \frac{\pi^{2} h^{2} j^{2}}{\sin ^{2}(\pi h j)}\right) \sum_{|j| \leq k} \frac{4 \sin ^{2}(\pi h j)}{h^{2}}\left|\beta(f)_{j}\right|^{2} \\
& =\left(\sup _{|x| \leq \frac{1}{2}} \frac{\pi x}{\sin \pi x}\right)^{2} \sum_{|j| \leq k}\left|\frac{\mathrm{e}^{2 \pi i h j}-1}{h} \beta(f)_{j}\right|^{2} \\
& \leq \frac{\pi^{2}}{4}\left\|\Delta_{h} \mathbb{P}_{k} f\right\|_{2}^{2} .
\end{aligned}
$$

Therefore

$$
\left\|\partial_{x} \mathbb{P}_{k} f\right\|_{2} \leq \frac{\pi}{2}\left\|\partial_{x} f\right\|_{2}
$$

Since $f-\mathbb{P}_{k} f$ is zero on all collocation points $x_{m}$, Poincaré inequality on the intervals $\left(x_{m}, x_{m+1}\right)$ implies that

$$
\left\|f-\mathbb{P}_{k} f\right\|_{2} \lesssim h\left\|\partial_{x}\left(f-\mathbb{P}_{k} f\right)\right\|_{2} .
$$

We may go deeper in the properties of the projection $\mathbb{P}_{k}$. In fact,

$$
\left\|\mathbb{P}_{k} f-f\right\|_{H^{1}}=o(1) .
$$

To see this, since the sequence $\mathcal{F} f$ is absolutely integrable, we have

$$
\begin{aligned}
\beta(f)_{j} & =h \sum_{|m| \leq k} f\left(x_{m}\right) \mathrm{e}^{-2 \pi \mathrm{i} j x_{m}} \\
& =h \sum_{|m| \leq k}\left(\sum_{\ell \in \mathbb{Z}} \mathcal{F} f(\ell) \mathrm{e}^{2 \pi \mathrm{i} \ell x_{m}}\right) \mathrm{e}^{-2 \pi \mathrm{i} j x_{m}} \\
& =h \sum_{|m| \leq k} \sum_{\ell \in \mathbb{Z}} \mathcal{F} f(\ell) \mathrm{e}^{2 \pi \mathrm{i}(\ell-j) x_{m}} \\
& =h \sum_{\ell \in \mathbb{Z}} \mathcal{F} f(\ell) \sum_{|m| \leq k} \mathrm{e}^{2 \pi \mathrm{i}(\ell-j) x_{m}},
\end{aligned}
$$


and the summation over $m$ vanishes except when $\ell \equiv j$ (i.e. $\ell-j$ is divisible by $\left.h^{-1}=2 k+1\right)$. Hence

$$
\beta(f)_{j}=\mathcal{F} \mathbb{P}_{k} f(j)=\sum_{\substack{\ell \in Z \\ \ell \equiv j}} \mathcal{F} f(\ell), \quad \text { for }|j| \leq k .
$$

We conclude that

$$
\frac{1}{4 \pi^{2}}\left\|\partial_{x}\left(f-\mathbb{P}_{k} f\right)\right\|_{2}^{2}=\sum_{|j| \leq k} j^{2}\left|\sum_{\substack{\ell \equiv j \\ \ell \neq j}} \mathcal{F} f(\ell)\right|^{2}+\sum_{|j|>k} j^{2}|\mathcal{F} f(j)|^{2} .
$$

But

$$
\begin{aligned}
\sum_{|j| \leq k} j^{2}\left|\sum_{\substack{\ell \equiv j \\
\ell \neq j}} \mathcal{F} f(\ell)\right|^{2} & \leq \sum_{|j| \leq k} j^{2}\left(\sum_{\substack{\ell \equiv j \\
\ell \neq j}} \frac{1}{\ell^{2}}\right)\left(\sum_{\substack{\ell \equiv j \\
\ell \neq j}} \ell^{2}|\mathcal{F} f(\ell)|^{2}\right) \\
& \leq\left(\sup _{|j| \leq k} \sum_{\substack{\ell \equiv j \\
\ell \neq j}} \frac{j^{2}}{\ell^{2}}\right) \sum_{|j| \leq k} \sum_{\substack{\ell \equiv j \\
\ell \neq j}} \ell^{2}|\mathcal{F} f(\ell)|^{2} \\
& \leq\left(\sup _{\substack{|j| \leq k \\
\sum_{\ell \neq 0}}}\left(\frac{j}{h^{-1} \ell+j}\right)^{2}\right) \sum_{|\ell|>k} \ell^{2}|\mathcal{F} f(\ell)|^{2} .
\end{aligned}
$$

On the other hand, since $h^{-1} j^{-1}<2$ for $|j| \leq k$,

$$
\sum_{\ell \neq 0}\left(\frac{1}{h^{-1} j^{-1} \ell+1}\right)^{2}<\sum_{\ell \neq 0} \frac{1}{(2|\ell|-1)^{2}}=\frac{\pi^{2}}{4}
$$

Therefore

$$
\left\|\partial_{x}\left(f-\mathbb{P}_{k} f\right)\right\|_{2} \rightarrow 0 .
$$

It is noteworthy that for $f \in H^{s}, s \geq 1$ we have

$$
\left\|\partial_{x}\left(f-\mathbb{P}_{k} f\right)\right\|_{2}=o\left(k^{1-s}\right) \text {. }
$$

Now we are able to state the following proposition.

Proposition 4.3. If we extend $\mathbb{P}_{k}$ to act component-wise on the elements of $H$, then the hypotheses of theorem (4.1) are satisfied with $\omega_{k}=h=(2 k+1)^{-1}$.

Remark 4.4. Note that theorem (4.1) states that the finite difference of the numerical solution converges to the derivative of the actual solution.

Remark 4.5. Although we have used for simplicity $h=1 /(2 k+1)$ for the theoretical proofs, we will use $h=2^{-k_{0}}$ in the simulations instead since the FFT algorithm is faster for the powers of two. There is no need to say that the theoretical proof of convergence can be written upon slightly modifying the above arguments. 
We translate the system (4.3) in the language of DFT. Let us denote the DFT of $\mathbf{f}^{(k)}$ by $\mathbf{g}$, i.e. $\mathbf{f}^{(k)}=\widetilde{\mathbf{g}}$. Then the system (4.3) reads

$$
\mathbf{S}_{t} \partial_{t} \mathbf{S}_{-t} \widetilde{\mathbf{g}}=\mathbb{P}_{k} \mathbf{N} \widetilde{\mathbf{g}} \text {. }
$$

This is equivalent to say that the equality holds at each collocation point $x_{m}$ for $|m| \leq k$. Hence recalling the fact that $\widetilde{\mathbf{g}} \in E_{k}$, the above system could be written as

$$
\mathcal{S}_{v t} \frac{\mathrm{d}}{\mathrm{d} t} \mathcal{S}_{-v t} \mathbf{g}_{v}=(N \mathbf{g})_{v}:=[v \mathcal{H} n]_{-} n-\left(\mu_{+}[\mathcal{H} n]_{-}+\mu_{-}[\mathcal{H} n]_{+}\right) \mathbf{g}_{v} .
$$

where $\mathcal{H}$ and $\mathcal{S}$ mean the discrete versions of the Hilbert and the shift operators respectively and $n=\sum_{v} \mathbf{g}_{v}$.

Proposition 4.6. For the solution to the system (4.11) we have

$$
\sum_{|m| \leq k} n_{m}=\sum_{|m| \leq k} n_{0 m}
$$

Proof. If we take the sum of (4.11) over $v$, the right hand side vanishes and hence

$$
0=\sum_{m} \sum_{v}\left(\mathcal{S}_{v t} \frac{\mathrm{d}}{\mathrm{d} t} \mathcal{S}_{-v t} \mathbf{g}_{v}\right)_{m}=\sum_{v} \sum_{m}\left(\mathcal{S}_{v t} \frac{\mathrm{d}}{\mathrm{d} t} \mathcal{S}_{-v t} \mathbf{g}_{v}\right)_{m}
$$

For any vector $b$, recalling the definition of the discrete shift operator (4.6), we have

$$
\sum_{m}\left(\mathcal{S}_{y} b\right)_{m}=\left(\mathcal{F}_{\mathrm{d}} \mathcal{S}_{y} b\right)_{j=0}=\left(\mathcal{F}_{\mathrm{d}} b\right)_{j=0}=\sum_{m} b_{m}
$$

Therefore

$$
\begin{aligned}
0 & =\sum_{v} \sum_{m}\left(\frac{\mathrm{d}}{\mathrm{d} t} \mathcal{S}_{-v t} \mathbf{g}_{v}\right)_{m}=\sum_{v} \frac{\mathrm{d}}{\mathrm{d} t} \sum_{m}\left(\mathcal{S}_{-v t} \mathbf{g}_{v}\right)_{m} \\
& =\sum_{v} \frac{\mathrm{d}}{\mathrm{d} t} \sum_{m} \mathbf{g}_{v, m}=\frac{\mathrm{d}}{\mathrm{d} t} \sum_{m} \sum_{v} \mathbf{g}_{v, m}=\frac{\mathrm{d}}{\mathrm{d} t} \sum_{m} n_{m}
\end{aligned}
$$

which completes the proof.

Now we are able to solve this system of ODE's with the vector $\mathbf{S}_{-t} \mathbf{g}$ as the unknown, using any familiar method. It is not difficult to see that any explicit Runge-Kutta method is equivalent to splitting the problem into separate steps for transport and reaction. In the simplest framework if one chooses the explicit Euler method, the problem is reduced to the calculation of the nonlinearity first and then to transport the corrected densities. Note that we do not need any CFL condition for the stability of such methods. We implement Heun's method [22]. If we denote by $\tau$ the time step, this method reads as follows,

$$
\mathbf{g}(t+\tau)=\mathbf{S}_{\tau} \mathbf{g}(t)+\frac{\tau}{2}\left(\mathbf{S}_{\tau} N \mathbf{g}(t)+N \mathbf{S}_{\tau}(\mathbf{g}(t)+\tau N \mathbf{g}(t))\right) .
$$

Also one may choose the ratio $\tau / h$ an integer number so that the shift operator is a simple shift of the samples. 


\section{Blow-up and numerical simulation}

Here we concentrate on the case $V=\{+1,-1\}$, i.e. the cells may move to left or right with constant velocity. Let us rewrite the equations in terms of $n=p+q$ and $j=p-q$,

$$
\left\{\begin{array}{l}
\partial_{t} n+\partial_{x} j=0 \\
\partial_{t} j+\partial_{x} n=-n \mathbb{H} n-j|\mathbb{H} n| .
\end{array}\right.
$$

On the circle. Consider $I(t)=\int n(x) \cos 2 \pi x \mathrm{~d} x$. If $\alpha=0$,

$$
\begin{aligned}
\frac{\mathrm{d}}{\mathrm{d} t} I= & -\int \partial_{x} j(x) \cos 2 \pi x \mathrm{~d} x=2 \pi \int j(x) \sin 2 \pi x \mathrm{~d} x . \\
\frac{1}{2 \pi} \frac{\mathrm{d}^{2}}{\mathrm{~d} t^{2}} I= & -\int\left(\partial_{x} n+n \mathbb{H} n+j|\mathbb{H} n|\right) \sin 2 \pi x \mathrm{~d} x \\
= & 2 \pi \int n(x) \cos 2 \pi x \mathrm{~d} x \\
& -\frac{1}{2} \iint(\sin 2 \pi x-\sin 2 \pi y) \cot \pi(x-y) n(x) n(y) \mathrm{d} x \mathrm{~d} y \\
& -\int j|\mathbb{H} n| \sin 2 \pi x \mathrm{~d} x \\
= & 2 \pi I-\frac{1}{2} \int(\cos 2 \pi x+\cos 2 \pi y) n(x) n(y) \mathrm{d} x \mathrm{~d} y \\
& -\int j|\mathbb{H} n| \sin 2 \pi x \mathrm{~d} x \\
= & (2 \pi-M) I-\int j|\mathbb{H} n| \sin 2 \pi x \mathrm{~d} x .
\end{aligned}
$$

On the whole space. We continue by analyzing the second momentum of the density, i.e. $I(t)=\frac{1}{2} \int x^{2} n(t, x) \mathrm{d} x$, in the case $\alpha=0$,

$$
\begin{aligned}
\frac{\mathrm{d}}{\mathrm{d} t} I & =\frac{1}{2} \int x^{2} \partial_{t} n=-\frac{1}{2} \int x^{2} \partial_{x} j=\int x j . \\
\frac{\mathrm{d}^{2}}{\mathrm{~d} t^{2}} I & =\int x \partial_{t} j \\
& =-\int x \partial_{x} n-\int x n \mathbb{H} n-\int x j|\mathbb{H} n| \\
& =M-\frac{M^{2}}{2 \pi}-\int x j|\mathbb{H} n| .
\end{aligned}
$$

These inequalities suggests that maybe the critical mass is $M=2 \pi$, the same as the modified macroscopic Keller-Segel model (see [7]). Unfortunately, at this point we are unable to prove a theoretical blow-up result, but numerical results suggest that this conjecture is true.

We have generated the figures in this article using MATLAB software. In figure (1), we have two equal masses concentrated at two points. At initial time, half of the cells are moving to the right and half of them to the left. One 


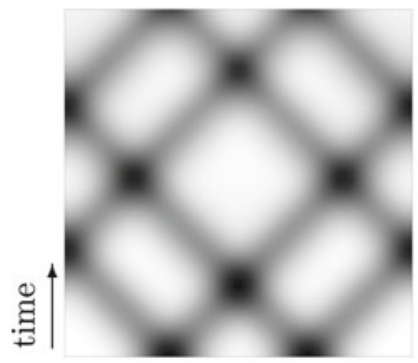

(a) $M=0.2 \pi$

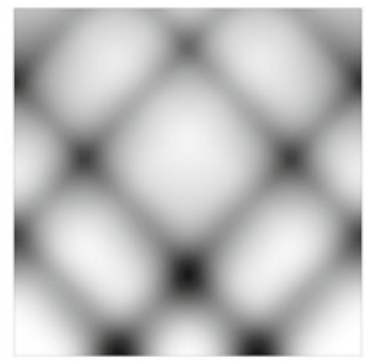

(b) $M=0.8 \pi$

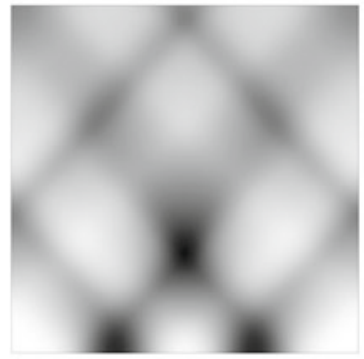

(c) $M=1.6 \pi$

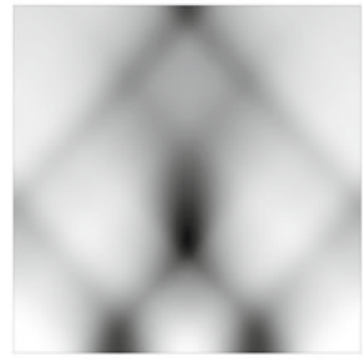

(d) $M=2.0 \pi$

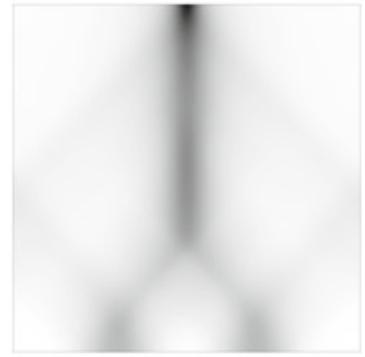

(e) $M=2.4 \pi$

FiguRE 1. Here $p_{0}=q_{0}=C_{M}\left(\exp \left(-100(x-0.2)^{2}\right)+\right.$ $\left.\exp \left(-100(x+0.2)^{2}\right)\right)$ as the initial data and the constant $C_{M}$ is such that the total mass equals to $M$. One can see the evolution of cells density corresponding to $h=2^{-14}$ and $\tau / h=2$ until time $T=1.0$ in the case $\alpha=0$

could see that for very small masses, each part of the mass is separated into two parts which move to the right or to the left and in every collision point, they will cross each other without much effect. For larger masses, the cells start to spread in the medium. For $M>2 \pi$, but not too large, the aggregation between cells overcome the transport and only a small portion of the cells scape from concentrating at the center. If $M$ is large enough, blow-up occur at the center.

The difference between figure (1) and figure (2) is that in the latter the cells are moving towards each other at the initial time. This suggests that the blow-up may occur for smaller masses than the above mentioned situation.

In figure (3), we put a larger portion of the mass at the right. It is seen that the larger mass absorbs the smaller one for large enough total masses. Another interesting observation in this situation is that after a short period of time, the total mass of the particles which move to the right becomes almost equal to the mass of ones which move to the left.

In figure (4), we start from a Gaussian distributed mass around the center. One can see the evolution of the system for different amounts of masses. 


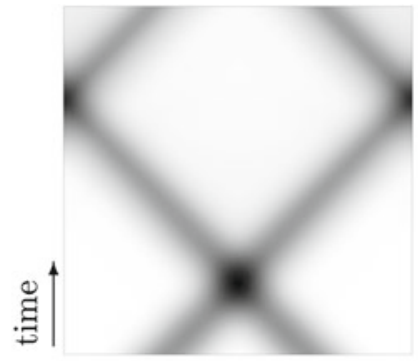

(a) $M=0.2 \pi$

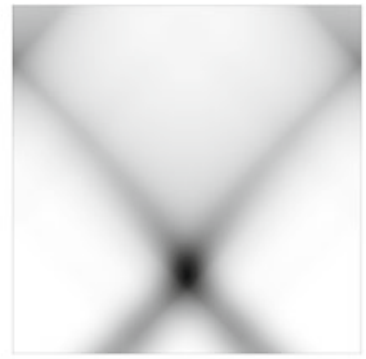

(b) $M=0.8 \pi$

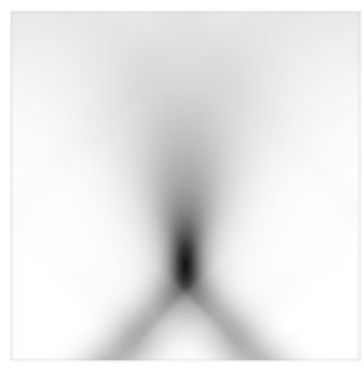

(c) $M=1.4 \pi$

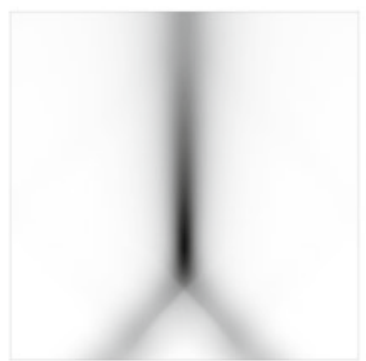

(d) $M=1.8 \pi$

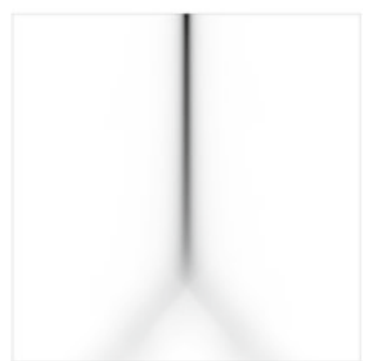

(e) $M=2.1 \pi$

Figure 2. Here the initial data are $p_{0}=C_{M} \exp (-100(x+$ $\left.0.2)^{2}\right), \quad q_{0}=C_{M} \exp \left(-100(x-0.2)^{2}\right)$, and the constant $C_{M}$ is such that the total mass equals to $M$. The parameters $h, \tau$ and $T$ are as in fig. (1)

If we consider the case $\alpha>0$, it is observed that the critical mass is greater for larger $\alpha$.

Also we may ask a question here to understand the possible profile of the blow-up in $\mathbb{R}$. Note that this system is invariant under the scaling $u_{\lambda}(t, x)=$ $\lambda u(\lambda t, \lambda x)$. So it is reasonable to try to find a solution to the backward problem of the form $n(t, x)=\frac{1}{t} n_{*}\left(\frac{x}{t}\right)$ and $j(t, x)=\frac{1}{t} j_{*}\left(\frac{x}{t}\right)$. If one let $y=\frac{x}{t}$, simple calculations yield

$$
\left\{\begin{array}{l}
\left(y n_{*}\right)^{\prime}+j_{*}^{\prime}=0 \\
\left(y j_{*}\right)^{\prime}+n_{*}^{\prime}=-n_{*} \mathcal{H} n_{*}-j_{*}\left|\mathcal{H} n_{*}\right| .
\end{array}\right.
$$

If we assume some integrability assumption on $n_{*}$ and $j_{*}$, the first equation implies $j_{*}=-y n_{*}$. Hence we end up with the following equation for $n_{*}$,

$$
\left(\left(1-y^{2}\right) n_{*}\right)^{\prime}+n_{*} \mathcal{H} n_{*}-y n_{*}\left|\mathcal{H} n_{*}\right|=0, \quad-1 \leq y \leq 1 .
$$

Note that since $|j| \leq n$, so $n_{*}$ must be zero outside the interval $[-1,1]$.

Theorem 5.1. If $\alpha=0$, there is no nonnegative weak solution to (5.2) in $L^{2}$ provided that $0<M \leq 2 \pi$ where $M=\int n_{*}$. 


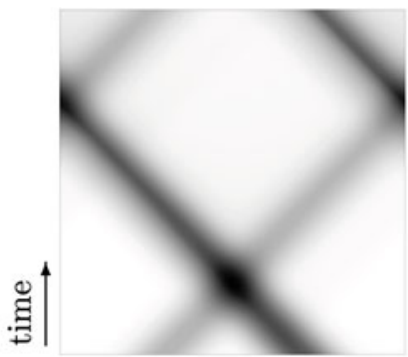

(a) $M=0.2 \pi$

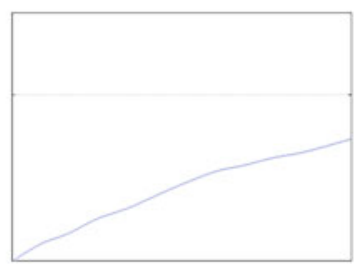

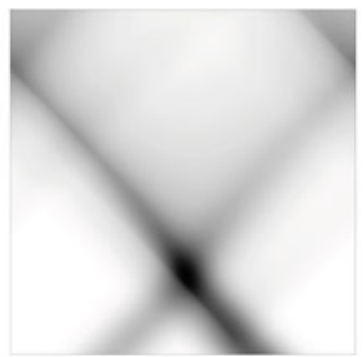

(b) $M=0.8 \pi$

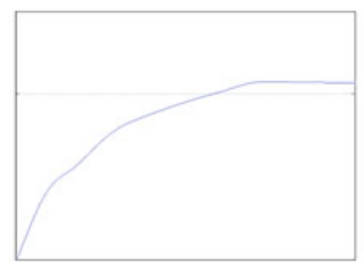

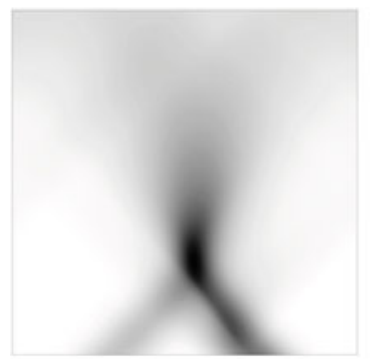

(c) $M=1.4 \pi$

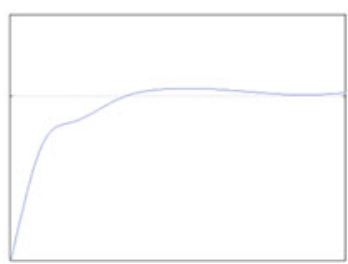

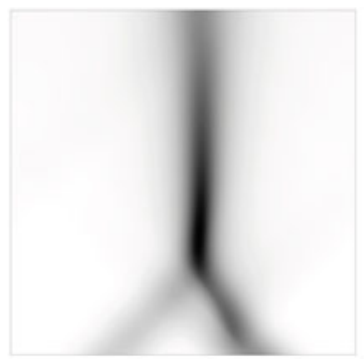

(d) $M=1.8 \pi$

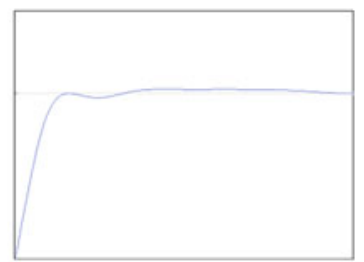

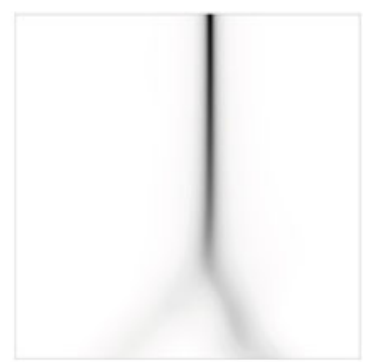

(e) $M=2.1 \pi$

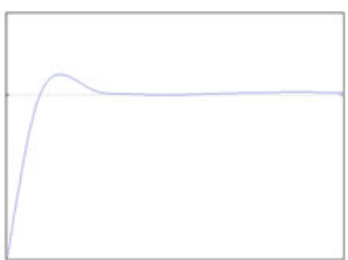

Figure 3. We set $p_{0}=0.3 C_{M} \exp \left(-100(x+0.2)^{2}\right)$ and $q_{0}=$ $0.7 C_{M} \exp \left(-100(x-0.2)^{2}\right)$ and the constant $C_{M}$ is such that the total mass equals to $M$. The parameters $h, \tau$ and $T$ are as in fig. (1). You can see also the evolution of the ratio $\int p / M$. The $x$-axis indicates the time. The $y$-axis indicates this ratio and it is scaled to the interval $[0.3,0.6]$

Proof. Assume by the way of contradiction that the function $n$ which is supported in $[-1,1]$ is a solution (we rename the unknown again for simplicity). Therefore if we multiply the equation by $y$ and integrate it, we obtain

$$
-M+\int y^{2} n+\frac{M^{2}}{2 \pi}-\int y^{2} n|\mathbb{H} n|=0 .
$$




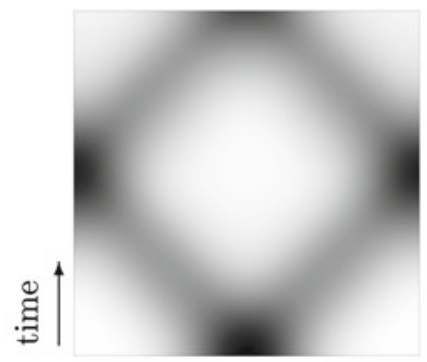

(a) $M=0.2 \pi$

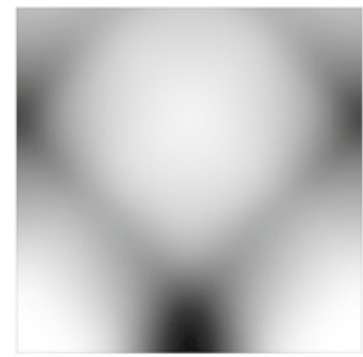

(b) $M=0.8 \pi$

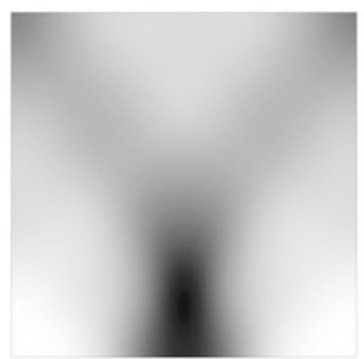

(c) $M=1.4 \pi$

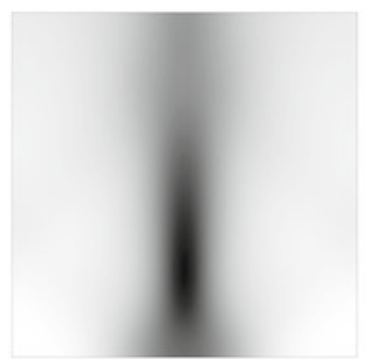

(d) $M=1.8 \pi$

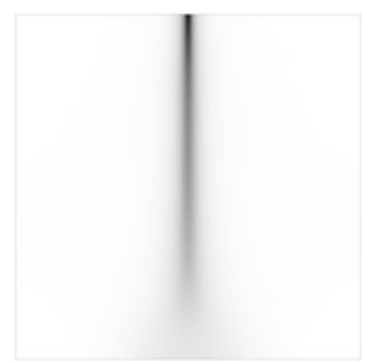

(e) $M=2.2 \pi$

FigurE 4. Starting with $p_{0}=q_{0}=C_{M} \exp \left(-30 x^{2}\right)$

On the other hand, we may compute

$$
\begin{aligned}
2 \pi & \int y^{2} n|\mathbb{H} n| \\
\geq & 2 \pi \int y|y| n \mathbb{H} n \\
& =\iint \frac{x|x|-y|y|}{x-y} n(x) n(y) \mathrm{d} x \mathrm{~d} y \\
& =\iint_{x y>0}(|x|+|y|) n(x) n(y) \mathrm{d} x \mathrm{~d} y+\iint_{x y<0} \frac{x^{2}+y^{2}}{|x|+|y|} n(x) n(y) \mathrm{d} x \mathrm{~d} y \\
\geq & 2 \iint_{x y>0}|y| n(x) n(y) \mathrm{d} x \mathrm{~d} y+\iint_{x y<0} \frac{|x|+|y|}{2} n(x) n(y) \mathrm{d} x \mathrm{~d} y \\
= & 2 \iint_{x y>0}|y| n(x) n(y) \mathrm{d} x \mathrm{~d} y+\iint_{x y<0}|y| n(x) n(y) \mathrm{d} x \mathrm{~d} y \\
> & M \int|y| n .
\end{aligned}
$$


Therefore

$$
\begin{aligned}
\frac{M^{2}}{2 \pi}-M+\int|y| n & \geq \frac{M^{2}}{2 \pi}-M+\int y^{2} n \\
& =\int y^{2} n|\mathbb{H} n| \\
& >\frac{M}{2 \pi} \int|y| n .
\end{aligned}
$$

Hence

$$
\left(\frac{M}{2 \pi}-1\right)\left(M-\int|y| n\right)>0,
$$

which contradicts the assumption $M \leq 2 \pi$, since $\int|y| n \leq M$.

\section{Acknowledgments}

It is a pleasure for the author to thank Dr. Vincent Calvez (ENS, Lyon) for having suggested the study of this problem. The author is grateful to Otared Kavian, François Murat and Luc Tartar for insights and helpful discussions regarding the properties of the nonlinear operator $\varphi \longmapsto[\varphi]_{+}$in the Sobolev spaces $H^{s}$ (lemma 2.11). This paper is part of the author's research in the period of his $\mathrm{PhD}$ studies, in the framework of a joint supervision by Prof. Mahmoud Hesaraki (SUT) and Prof. Otared Kavian (UVSQ), as the PhD advisors. The author is indebted to financial supports from "Sharif University of Technology" and the cultural section of French embassy in Tehran, Iran. (The "Irano-European Master of Mathematics" is supported by the French government and Université de Versailles Saint Quentin).

\section{References}

[1] Adams, R.A.: Sobolev spaces. Academic Press (A subsidiary of Harcourt Brace Jovanovich, Publishers), New York, London. Pure Appl. Math. 65 (1975)

[2] Blanchet, A., Dolbeault, J., Perthame, B.: Two-dimensional Keller-Segel model: optimal critical mass and qualitative properties of the solutions. Electron. J. Diff. Equ. pages no. 44, 32 pp. (electronic) (2006)

[3] Bournaveas, N., Calvez, V.: Global existence for the kinetic chemotaxis model without pointwise memory effects, and including internal variables. Kinet. Relat. Models 1(1), 29-48 (2008)

[4] Bournaveas, N., Calvez, V.: Critical mass phenomenon for a chemotaxis kinetic model with spherically symmetric initial data. Ann. Inst. H. Poincaré Anal. Non Linéaire 26(5), 1871-1895 (2009)

[5] Bournaveas, N., Calvez, V., Gutiérrez, S., Perthame, B.: Global existence for a kinetic model of chemotaxis via dispersion and Strichartz estimates. Comm. Partial Diff. Equ. 33(1-3), 79-95 (2008) 
[6] Calvez, V., Corrias, L.: The parabolic-parabolic Keller-Segel model in $\mathbb{R}^{2}$. Commun. Math. Sci. 6(2), 417-447 (2008)

[7] Calvez, V., Perthame, B., Sharifi tabar, M.: Modified Keller-Segel system and critical mass for the log interaction kernel. In: Stochastic analysis and partial differential equations, volume 429 of Contemp. Math., pp. 45-62. Amer. Math. Soc., Providence, RI (2007)

[8] Chalub, F., Dolak-Struss, Y., Markowich, P., Oelz, D., Schmeiser, C., Soreff, A.: Model hierarchies for cell aggregation by chemotaxis. Math. Models Methods Appl. Sci. 16(7 Suppl), 1173-1197 (2006)

[9] Chalub, F.A.C.C., Markowich, P.A., Perthame, B., Schmeiser, C.: Kinetic models for chemotaxis and their drift-diffusion limits. Monatsh. Math. 142(1-2), 123-141 (2004)

[10] Chalub, F.A.C.C., Rodrigues, J.F.: A class of kinetic models for chemotaxis with threshold to prevent overcrowding. Port. Math. (NS) 63(2), 227-250 (2006)

[11] Corrias, L., Perthame, B., Zaag, H.: Global solutions of some chemotaxis and angiogenesis systems in high space dimensions. Milan J. Math. 72, 1-28 (2004)

[12] Dickinson, R.B., Tranquillo, R.T.: A stochastic model for cell random motility and haptotaxis based on adhesion receptor fluctuations. J. Math. Biol. 31, 563-600 (1993)

[13] Erban, R., Othmer, H.G.: From individual to collective behavior in bacterial chemotaxis. SIAM J. Appl. Math. 65(2), 361-391 (electronic) (2004/2005)

[14] Erban, R., Othmer, H.G.: From signal transduction to spatial pattern formation in E. coli: a paradigm for multiscale modeling in biology. Multiscale Model. Simul. 3(2), 362-394 (electronic) (2005)

[15] Erban, R., Othmer, H.G.: Taxis equations for amoeboid cells. J. Math. Biol. 54(6), 847-885 (2007)

[16] Hillen, T.: $\mathrm{M}^{5}$ mesoscopic and macroscopic models for mesenchymal motion. J. Math. Biol. 53(4), 585-616 (2006)

[17] Hwang, H.J., Kang, K., Stevens, A.: Global solutions of nonlinear transport equations for chemosensitive movement. SIAM J. Math. Anal. 36(4), 1177-1199 (electronic) (2005)

[18] Jäger, W., Luckhaus, S.: On explosions of solutions to a system of partial differential equations modelling chemotaxis. Trans. Amer. Math. Soc. 329(2), 819-824 (1992)

[19] Keller, E.F., Segel, L.A.: Initiation of slime mold aggregation viewed as an instability. J. Theor. Biol. 26, 399-415 (1970)

[20] Othmer, H.G., Dunbar, S.R., Alt, W.: Models of dispersal in biological systems. J. Math. Biol. 26(3), 263-298 (1988) 
[21] Othmer, H.G., Hillen, T.: The diffusion limit of transport equations. II. Chemotaxis equations. SIAM J. Appl. Math. 62(4), 1222-1250 (electronic) (2002)

[22] Stoer, J., Bulirsch, R.: Introduction to numerical analysis, volume 12 of Texts in Applied Mathematics. 3rd edn. Springer, New York, 2002 (Translated from the German by R. Bartels, W. Gautschi and C. Witzgall)

Mohsen Sharifi tabar

Sharif University of Technology, Azadi av.,

P.O. Box 11365-9415,

Tehran,

Iran

e-mail: sharifitabar@math.sharif.ir, sharifitabar@math.uvsq.fr

and

Lab. de Math. de Versailles (LMV-UMR8100),

UVSQ, 45, av. des Ètats-Unis,

78035, Versailles cedex,

France.

Received: 22 June 2010 .

Revised: 21 September 2010.

Accepted: 01 October 2010. 